\title{
Chemostratigraphy of the early Pliocene diatomite interval from MIS AND-1B core (Antarctica): Palaeoenvironment implications
}

\author{
Giovanna Scopelliti a,*, Adriana Bellanca a, Donata Monien ${ }^{\text {b }}$, Gerhard Kuhn ${ }^{\mathrm{c}}$ \\ a Dipartimento di Scienze della Terra e del Mare (DiSTeM), Università di Palermo, Via Archirafi 36, 90123 Palermo, Italy \\ b Microbiogeochemistry, Institute for Chemistry and Biology of the Marine Environment, Carl-von-Ossietzky University, Oldenburg, Germany \\ c Geosciences Division, Alfred Wegener Institute for Polar and Marine Research, Bremerhaven, Germany
}

\section{A R T I C L E I N F O}

\section{Article history}

Received 12 June 2012

Accepted 15 January 2013

Available online 23 January 2013

\section{Keywords:}

biogenic opal

element geochemistry

palaeoenvironment

\begin{abstract}
A B S T R A C T
The AND-1B drill core (1285 m-long) was recovered, inside the ANDRILL (ANtarctic geological DRILLing) Program, during the austral summer of 2006/07 from beneath the floating McMurdo Ice Shelf. Drilling recovered a stratigraphic succession of alternating diamictites, diatomites and volcaniclastic sediments spanning about the last $14 \mathrm{Ma}$. A core portion between 350 and $480 \mathrm{mbsf}$, including a $80 \mathrm{~m}$-thick diatomite interval recording the early Pliocene warming event, was investigated in term of opal biogenic content and element geochemistry. Across the diatomite interval, in spite of the lithological uniformity, a fluctuating biogenic opal profile mirrors the $\delta^{18} \mathrm{O}$ record, testifying a decrease in productivity when temperature drops as a consequence of small glacial fluctuations. The comparison of biogenic opal data with Chaetoceros spp. abundances from Konfirst et al. (2012) documents alternations between periods of high primary productivity in stratified surface waters and of enhanced terrigenous input in ice-free conditions. Cluster analysis discriminates elements associated to terrigenous input from those subject to biogenic control. Further separation in sub-cluster was interpreted in term of different element response to changes in provenance but also to depositional/early diagenetic conditions at the seafloor. Whilst $\mathrm{K}$ and $\mathrm{Ti}$ are related to different sediment sources confirming previous studies from the same interval, $\mathrm{V}, \mathrm{Zn}$ and, to a lesser extent, Fe, document reducing/anoxic conditions during the diatomites deposition (in particular in 400-460 mbsf interval). $\mathrm{Mg}$, $\mathrm{Sr}$ and $\mathrm{Mn}$ contents are related to authigenic carbonate precipitation whilst Ba is interested by nonsteady-state processes leading to local peaks of barium below the sulphate-rich/sulphate-poor pore water boundary where generally the low degree of barite saturation is responsible for Ba remobilization. Such alteration in depositional dynamics, responsible of the precipitation of an oxygen-depleted barium phase, was probably induced by change in sedimentation rate and/or in palaeoenvironmental conditions.
\end{abstract}

(c) 2013 Elsevier B.V. All rights reserved.

\section{Introduction}

The warm early Pliocene event ( 4.5 to 3.0 million years ago) is considered the most recent interval with a climate warmer than today and for this reason is widely studied as an analogue for the effects of human-induced warming (e.g. Barron, 1992; Cronin et al., 1993; Dowsett et al., 1996; Haywood and Valdes, 2004; Dowsett et al., 2005; Pollard and DeConto, 2009). During much of the Pliocene, the Ross Sea and other Antarctic coastal regions experienced marine biogenic production on the scale of that occurring today in settings closer to modern sub-polar coastal regions (Winter et al., 2010). Recently, a thick diatomite interval $(\sim 80 \mathrm{~m})$ recovered within the AND-1B drill core (Western Ross Sea) has offered a unique opportunity to study the Antarctica near shore environment during the early Pliocene warm period. High-resolution investigation on diatom assemblages and carbonate stable isotopes highlighted the Pliocene warming climax

\footnotetext{
* Corresponding author. Tel.: + 39091 23861622; fax: + 390916168376.

E-mail address: giovanna.scopelliti@unipa.it (G. Scopelliti).
}

and a strong influence of the orbitally-controlled cyclicity within a so uniform lithological unit (Konfirst et al., 2011; Scopelliti et al., 2011; Konfirst et al., 2012). Different portions of AND-1B core (Giorgetti et al., 2009; Monien et al., 2012), including the diatomite interval between 380 to 460 mbsf (Konfirst et al., 2012), have been the object of mineralogical and/or elemental geochemistry studies in order to assess major changes in sediment provenance within the McMurdo Sound and the role of material transport processes. Nevertheless, a detailed geochemical study of the $\sim 80 \mathrm{~m}$-thick diatomite interval aimed to the definition of deposition conditions is today lacking despite it can provide important information about a significant warming event in an ice proximal sedimentary sequence.

Sediments underlying areas of high biogenic productivity commonly exhibit, beside a strong increase in biogenic opal, peculiar trends in concentrations of some elements as $\mathrm{Ba}, \mathrm{V}, \mathrm{Zn}, \mathrm{Fe}$, that are strictly connected with variation in redox conditions at the seafloor. The link between enhanced surface productivity and increased amount of barium in the sediment is thought to be associated with decay of phytoplankton 
and inorganic precipitation of barite in water column or in supersaturated microenvironments at the seafloor (Dehairs et al., 1980; Bishop, 1988; Dehairs et al., 1991; von Breymann et al., 1992; Gingele and Dahmke, 1994; Paytan et al., 1996; Gingele et al., 1999; Ganeshram et al., 2003). On the other hand, under conditions of sulphate depletion in pore waters, which is usually encountered below oxygen-depleted bottom waters, the barite is dissolved and barium is released into the pore water (Brumsack and Gieskes, 1983; von Breymann et al., 1992; Gingele et al., 1999; McManus et al., 1999; Castellini et al., 2006; Scopelliti et al., 2006; Hendy, 2010; Henkel et al., 2012). In these conditions, increase of pH and carbonate alkalinity encourages the formation of dolomite and other authigenic carbonate (Vasconcelos and McKenzie, 1997; Warthmann et al., 2000; van Lith et al., 2003; Raiswell and Fisher, 2004; Scopelliti et al., 2009). Finally, redox-sensitive trace elements (as $\mathrm{V}$ and $\mathrm{Zn}$ ) tend to be more soluble under oxidizing conditions and very particle reactive under reducing conditions, resulting in authigenic enrichments of organic-decay related particles (e.g. Brumsack and Gieskes, 1983; Tribovillard et al., 2006). In this light, a comparative study of geochemical records and mineral associations can reveal depositional/early-diagenetic environments in which the presence of element enrichments associated with the precipitation of mineral phases can be indicative of specific Eh, pH or carbonate alkalinity conditions.

In this paper, high-resolution elemental geochemistry of the AND-1B Pliocene diatomite interval (350-480 mbsf) has been investigated. Fluctuations in biogenic opal signal have been correlated to benthic $\delta^{18}$ O LR04 stack (Lisiecki and Raymo, 2005) to evaluate the response of primary productivity to the small-scale glacial fluctuations and to calculate the sedimentation rate. Results have been compared with those obtained for same interval from Konfirst et al.
(2012). The relationship between terrigenous elements as potassium, titanium, zirconium and aluminium with vanadium, zinc, and iron is discussed in terms of variation in redox conditions at the sedimentwater interface as consequence of changes in the deposition conditions. Peaks in barium depth profile are interpreted as response to variation in deposition dynamics causing specific authigenic mineral associations.

\section{Setting}

The McMurdo Ice Shelf (MIS) drill site is located above a flexural moat basin adjacent to the Ross Island (Fig. 1). This basin formed in response to Quaternary crust loading by volcanoes of the Ross Island Massif, being superimposed on more regional subsidence associated with Neogene extension of the Terror Rift (Horgan et al., 2005; Naish et al., 2006; Fielding et al., 2008). This peculiar setting, favourable to the accumulation and preservation of thick silici- and volcani-clastic filling, permitted to recover a single, $1284.87 \mathrm{~m}$-deep, drill core (AND-1B) from the bathymetric and depocentral axis of the moat in $943 \mathrm{~m}$ of water from an ice shelf platform (Naish et al., 2007). The here investigated portion of MIS AND-1B core covers the interval between 350 and $480 \mathrm{~m}$ below seafloor (mbsf). Lithologically, it is characterized by the presence of the thicker $(84 \mathrm{~m})$ stratified diatomite interval with the age range between 4.7 and 3.4 Ma of the whole MIS AND-1B core interrupted, at $440 \mathrm{mbsf}$, by a "hiatus" of $\sim 800 \mathrm{kyr}$ (Naish et al., 2009). Other lithologies are represented by alternations of massive and stratified diamictite, sandstone and mudstone with dispersed clasts (Krissek et al., 2007). Chronostratigraphically, the

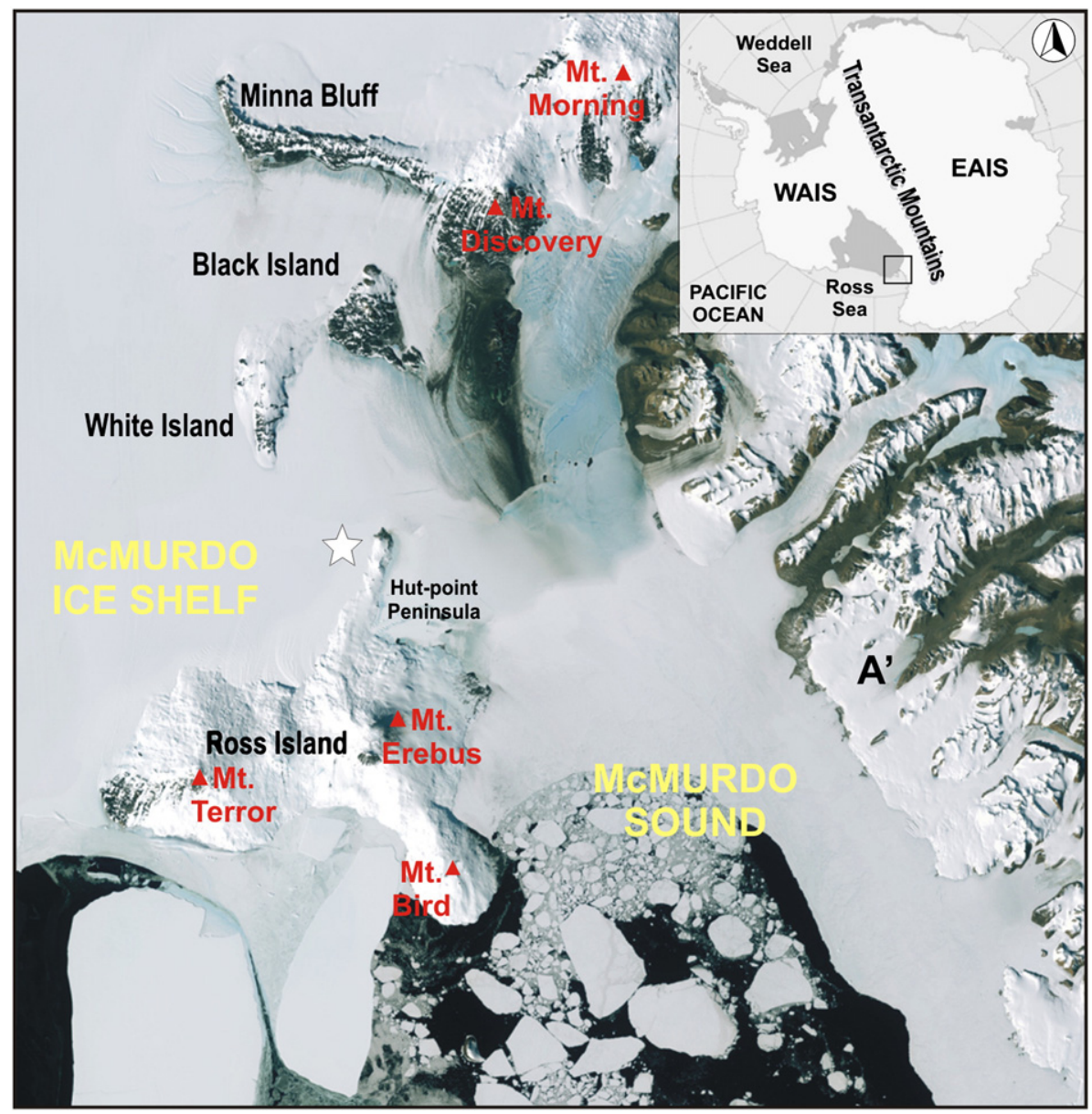

Fig. 1. Drill site MIS AND-1B (star) in the southeastern corner of the McMurdo Ice Shelf (image from USGS. International Polar Year 2007-2008). 
investigated sediments span the Zanclean/Piacenzian Planes (earlymiddle Pliocene; Scopelliti et al., 2011 and reference therein).

\section{Methodology}

\subsection{Analytical methods}

Major and minor element concentrations were determined on a total of 83 bulk-rock samples by X-ray fluorescence spectrometry (XRF) on pressed, boric-acid baked pellets, using a RIGAKU ZSX primus spectrometer. Data reduction was achieved using the method described by Franzini et al. (1975). Certified reference materials (BCR-1, BHVO-1, AGV-1) were used as monitors of data quality. Analytical errors were below 5\% for major elements, $\mathrm{Zr}$, and $\mathrm{Sr}$ and below $10 \%$ for $\mathrm{V}, \mathrm{Zn}, \mathrm{Rb}$, and $\mathrm{Ba}$. All samples were washed repeatedly in deionized water to avoid contamination due to presence of halite.

Biogenic silica was determined from 123 bulk sediment samples by the leaching method after Müller and Schneider (1993). For calculation of biogenic opal $10 \%$ for crystal water within opaline substances was added to the biogenic silica value. The analytical error of randomly choosen triplicate samples was between 2 and 5\%.

To investigate textural features of authigenic minerals, selected carbon-coated samples were observed under the Scanning Electron Microscope (SEM) LEO 440 with EDS system OXFORD ISIS and Si (Li) PENTAFET detector.

\subsection{Data analysis}

The relationships between major and minor elements are investigated through a data matrix that was preliminary standardized (Rock, 1988 ) in order to avoid pseudo correlation and artefacts due to variables quoted in different units in the multivariate analysis. The obtained data were treated with multivariate techniques of Cluster Analysis (CA) using STATISTICA data analysis package (version 6.0).

Cluster analysis was performed on the 83 sampling sites (Q-mode) and the 16 variables considered (R-mode) using the Ward's linkage method based on the Squared Euclidean distance which produces dendrograms with exceptionally well defined clusters.

\section{Results}

Biogenic opal as well as major and minor element concentrations for the investigated AND-1B core portion (350 to $480 \mathrm{mbsf}$ ) are listed in Table 1.

To better define the factors controlling the element geochemical behaviour in different core portions, CA was applied by processing the dataset both with respect to variables (elements) and cases (samples). The dendrogram in Fig. 2A defines two main clusters discriminating $\mathrm{Rb}, \mathrm{K}_{2} \mathrm{O}, \mathrm{Al}_{2} \mathrm{O}_{3}, \mathrm{Zn}, \mathrm{Zr}, \mathrm{Fe}_{2} \mathrm{O}_{3}, \mathrm{P}_{2} \mathrm{O}_{5}, \mathrm{~V}, \mathrm{TiO}_{2}$ (cluster $\mathrm{E} 1$ ), and $\mathrm{Ba}, \mathrm{MnO}$, $\mathrm{Sr}, \mathrm{CaO}, \mathrm{MgO}$, biogenic opal (cluster E2). Into the main clusters, sub-clusters E1a/E1b and E2a/E2b can be recognized. By processing geochemical data with respect to samples, clusters S1 and S2 and sub-clusters S1a and S1b were identified (Fig. 2B).

Depth profiles of $\mathrm{Al}_{2} \mathrm{O}_{3}, \mathrm{TiO}_{2}, \mathrm{CaO}$ and biogenic opal, chosen as representatives of elements falling into sub-clusters E1a, E1b, E2a, and E2b, respectively, are shown in Fig. 3.

From the bottom of the investigated core portion to $\sim 460 \mathrm{mbsf}$, $\mathrm{Al}_{2} \mathrm{O}_{3}$ and $\mathrm{TiO}_{2}$ show relatively high mean values (12.6\% and $0.9 \%$, respectively). Then, their concentrations decrease (markedly for $\mathrm{Al}$ ) fluctuating, throughout the $80 \mathrm{~m}$-thick diatomite interval, around mean values of 4.8 and $0.4 \%$, respectively, for $\mathrm{Al}_{2} \mathrm{O}_{3}$ and $\mathrm{TiO}_{2}$. Then, the curves of both elements exhibit an upcore increasing trend.

Depth profile of $\mathrm{CaO}$ shows a decrease throughout the diatomite interval with an average value of $1.2 \%$ after excluding three pronounced peaks at $425.60 / 426.66,437.10$, and $449.26 \mathrm{mbsf}(\mathrm{CaO}=13.5 / 15.9$, 25.2, 21.9\%, respectively; Fig. 3) that reflect Fe-dolomite-rich samples
(Scopelliti et al., 2011). In lithologies underlying the diatomite interval (below $460 \mathrm{mbsf}$ ) $\mathrm{CaO}$ percentages oscillate around 3.9\% whilst above the diatomite sequence the values gradually increase up to $7.3 \%$.

The biogenic opal curve appears as a mirror image of the $\mathrm{CaO}$ depth profile. Concentrations are on average $43.4 \%$ in the diatomite interval against an average value of $13.4 \%$ in other lithologies. Biogenic opal concentrations sharply decrease in correspondence of the three positive peaks of $\mathrm{CaO}$. Owing to the palaeoenvironmental significance of biogenic opal as proxy for diatom productivity, data from this work were superimposed on abundance values of Chaetoceros spp. reported in Konfirst et al. (2012).

\section{Discussion}

\subsection{Correlation of biogenic opal data to the oxygen isotope stage}

High resolution biogenic opal data are used to investigate the ice sheet behaviour during deposition of the thick diatomite interval from 360 to 460 mbsf. Increase of biogenic opal within the diatomite interval (Fig. 3) is strictly connected with the increase in primary productivity occurring during the early-middle Pliocene warm event (Grützner et al., 2005; Hillenbrand and Ehrmann, 2005; Hepp et al., 2006; Naish et al., 2007; Escutia et al., 2009; Winter et al., 2010, 2012). Lithological uniformity hinders the identification of orbital cyclicity and glacial variability within this extended period of high productivity open ocean environment. Naish et al. $(2007 ; 2009)$ used the iceberg-rafted debris (IBRD) as indicators of intervals in which outlet glaciers were still calving into the sea against periods in which the ice sheets retreated onto land. Scopelliti et al. (2011) found, within the thick diatomite interval, a relation between maxima in $\delta^{18} \mathrm{O}$ from the AND-1B bulk carbonate and those from the benthic LR04 (reported in Lisiecki and Raymo, 2005). Based on diatomite assemblages and Chaetoceros abundance, Konfirst et al. $(2011,2012)$ divided the diatomite interval into discrete diatom subunits. A comparison of the biogenic opal record (this paper) with the benthic $\delta^{18}$ O LR04 (Lisiecki and Raymo, 2005) shows that negative peaks in the biogenic opal curve correlate well with maxima in $\delta^{18} \mathrm{O}$ record (Fig. 4). This finding is consistent with that obtained by Konfirst et al. $(2011,2012)$ using siliceous microfossil data and testifies that relatively cold periods (higher oxygen isotopes; Lisiecki and Raymo, 2005) were associated to decrease in productivity. The correlation of biogenic opal data to the isotope record of Lisiecki and Raymo (2005) allowed us to constrain diverging sedimentation rates (SR) throughout the thick uniform diatomite interval (Fig. 4), falling in the ranges proposed by Konfirst et al. (2011). In particular, significant change in sedimentation rate are recorded in correspondence of two hiatus at $\sim 400$ and $\sim 455$ mbsf with values of 16.9, 8.7, and $29.3 \mathrm{~cm} / \mathrm{kyr}$ in $455-480,440-455$, and 350-440 mbsf intervals, respectively.

Interestingly, comparison with the Chaetoceros abundance curve from Konfirst et al. (2012) documents that intervals (365-385, 398-402, $425-440$, and 455-460 mbsf) in which the biogenic opal record correlates with that of Chaetoceros sp. alternate with intervals (385-398, 402-425 and 440-455 mbsf) characterized by wider excursions in biogenic opal concentrations with respect to Chaetoceros spp. (Fig. 3). This pattern is in accord with signals of seasonal diatom variability observed in Holocene deglacial sedimentary records from the Antarctic margin in response to changes in shelf waters (e.g. Maddison et al., 2005; Stickley et al., 2005; Leventer et al., 2006). These studies well document alternations between levels of diatom assemblage dominated by Chaetoceros spp. associated with stratified surface waters trapping nutrients and promoting high primary productivity, and levels of mixed open-water diatom assemblage associated with increased terrigenous input and related to ice-free, open water. 
Table 1

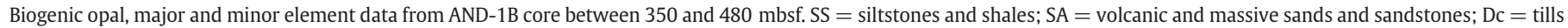
and diamictites; $\mathrm{D}=$ diatomites; dol = dolomite and dolomitic limestones.

\begin{tabular}{|c|c|c|c|c|c|c|c|c|c|c|c|c|c|c|c|c|c|}
\hline mbsf & Lithology & $\begin{array}{l}\text { Biog. opal } \\
\text { (wt.\%) }\end{array}$ & $\begin{array}{l}\mathrm{Si02} \\
\text { (wt.\%) }\end{array}$ & $\begin{array}{l}\text { Ti02 } \\
\text { (wt.\%) }\end{array}$ & $\begin{array}{l}\text { A1203 } \\
\text { (wt.\%) }\end{array}$ & $\begin{array}{l}\mathrm{P}_{2} \mathrm{O}_{5} \\
\text { (wt.\%) }\end{array}$ & $\begin{array}{l}\text { Fe203 } \\
\text { (wt.\%) }\end{array}$ & $\begin{array}{l}\text { MgO } \\
\text { (wt.\%) }\end{array}$ & $\begin{array}{l}\text { Mno } \\
\text { (wt.\%) }\end{array}$ & $\begin{array}{l}\mathrm{CaO} \\
\text { (wt.\%) }\end{array}$ & $\begin{array}{l}\text { K20 } \\
\text { (wt.\%) }\end{array}$ & $\begin{array}{l}\text { V } \\
(\mathrm{ppm})\end{array}$ & $\begin{array}{l}\mathrm{Zn} \\
(\mathrm{ppm})\end{array}$ & $\begin{array}{l}\mathrm{Rb} \\
\mathrm{ppm}\end{array}$ & $\begin{array}{l}\mathrm{Sr} \\
(\mathrm{ppm})\end{array}$ & $\begin{array}{l}\mathrm{Zr} \\
(\mathrm{ppm})\end{array}$ & $\begin{array}{l}\text { Ba } \\
(\mathrm{ppm})\end{array}$ \\
\hline 350.10 & Dc & 7 & 54.69 & 1.99 & 11.54 & 0.43 & 8.40 & 5.85 & 0.18 & 7.28 & 2.24 & 162 & 91 & 44 & 441 & 170 & 485 \\
\hline 351.68 & SA & 12 & 62.57 & 1.21 & 10.36 & 0.24 & 5.87 & 4.75 & 0.12 & 5.71 & 2.49 & 109 & 76 & 60 & 313 & 161 & 448 \\
\hline 353.98 & SS & 28 & 65.57 & 0.73 & 10.18 & 0.16 & 6.14 & 4.69 & 0.12 & 2.86 & 1.97 & 102 & 78 & 72 & 156 & 68 & 342 \\
\hline 354.96 & SS & 8 & 56.01 & 2.19 & 11.27 & 0.40 & 7.88 & 7.04 & 0.18 & 6.30 & 2.18 & 180 & 90 & 52 & 524 & 200 & 396 \\
\hline 356.26 & SS & 19 & 58.14 & 1.05 & 7.99 & 0.16 & 7.94 & 11.97 & 0.15 & 1.11 & 3.26 & 93 & 77 & 107 & 231 & 88 & 300 \\
\hline 356.56 & SA & 15 & 56.52 & 1.78 & 9.43 & 0.37 & 7.30 & 9.64 & 0.15 & 4.36 & 2.42 & 140 & 82 & 63 & 460 & 184 & 415 \\
\hline 359.12 & SS & 17 & 61.52 & 0.91 & 8.82 & 0.14 & 6.88 & 9.96 & 0.12 & 1.50 & 3.18 & 91 & 82 & 115 & 218 & 83 & 521 \\
\hline 360.06 & SS & 9 & & & & & & & & & & & & & & & \\
\hline 361.82 & SS & 23 & 58.35 & 1.47 & 10.75 & 0.23 & 7.10 & 7.20 & 0.13 & 4.51 & 2.67 & 145 & 86 & 68 & 309 & 115 & 387 \\
\hline 362.10 & SS & 25 & & & & & & & & & & & & & & & \\
\hline 362.92 & SS & 16 & & & & & & & & & & & & & & & \\
\hline 363.72 & Dc & 34 & 78.50 & 0.65 & 6.17 & 0.08 & 3.31 & 2.02 & 0.04 & 1.36 & 1.41 & 76 & 62 & 51 & 171 & 76 & 1706 \\
\hline 364.38 & $\mathrm{D}$ & 25 & 71.79 & 0.96 & 8.46 & 0.16 & 5.09 & 2.97 & 0.08 & 3.22 & 1.90 & 100 & 73 & 52 & 232 & 102 & 718 \\
\hline 366.32 & SS & 24 & 69.61 & 0.73 & 9.10 & 0.15 & 5.12 & 3.28 & 0.11 & 3.70 & 1.82 & 95 & 74 & 62 & 232 & 107 & 404 \\
\hline 367.94 & SS & 25 & 74.78 & 0.58 & 7.34 & 0.08 & 4.48 & 2.57 & 0.06 & 2.10 & 1.88 & 72 & 68 & 64 & 154 & 83 & 358 \\
\hline 370.24 & $\mathrm{Dc}$ & 18 & 71.14 & 0.85 & 8.24 & 0.13 & 5.49 & 3.20 & 0.08 & 3.01 & 2.01 & 97 & 71 & 61 & 208 & 101 & 384 \\
\hline 372.44 & Dc & 30 & 71.15 & 0.83 & 8.50 & 0.13 & 5.14 & 2.29 & 0.07 & 3.28 & 2.04 & 95 & 70 & 60 & 197 & 97 & 398 \\
\hline 372.84 & SS & 29 & & & & & & & & & & & & & & & \\
\hline 374.26 & SS & 27 & 72.63 & 0.59 & 7.95 & 0.11 & 4.59 & 2.81 & 0.10 & 3.13 & 1.64 & 83 & 70 & 55 & 179 & 75 & 377 \\
\hline 375.94 & Dc & 30 & & & & & & & & & & & & & & & \\
\hline 376.94 & $\mathrm{D}$ & 45 & 73.78 & 0.49 & 7.80 & 0.09 & 4.39 & 2.65 & 0.09 & 2.03 & 1.65 & 73 & 69 & 53 & 132 & 56 & 381 \\
\hline 378.16 & D & 38 & 78.49 & 0.40 & 7.00 & 0.06 & 3.41 & 2.12 & 0.08 & 1.19 & 1.50 & 65 & 61 & 49 & 100 & 56 & 333 \\
\hline 379.84 & D & 26 & 76.46 & 0.40 & 7.43 & 0.07 & 3.59 & 2.28 & 0.08 & 1.69 & 1.58 & 67 & 60 & 47 & 96 & 49 & 413 \\
\hline 380.96 & $\mathrm{D}$ & 36 & 74.21 & 0.47 & 7.94 & 0.10 & 3.97 & 2.51 & 0.09 & 2.29 & 1.64 & 72 & 64 & 51 & 138 & 73 & 483 \\
\hline 382.24 & D & 25 & 74.20 & 0.54 & 8.13 & 0.11 & 3.89 & 2.41 & 0.09 & 2.41 & 1.67 & 71 & 68 & 56 & 167 & 93 & 398 \\
\hline 383.06 & D & 43 & 78.46 & 0.32 & 6.48 & 0.04 & 3.15 & 2.05 & 0.07 & 0.75 & 1.38 & 56 & 59 & 49 & 74 & 40 & 270 \\
\hline 383.94 & D & 32 & 78.45 & 0.31 & 6.44 & 0.04 & 3.10 & 2.08 & 0.07 & 0.73 & 1.37 & 58 & 60 & 55 & 81 & 44 & 256 \\
\hline 385.04 & $\mathrm{D}$ & 25 & 68.29 & 0.37 & 10.10 & 0.08 & 5.23 & 3.59 & 0.10 & 1.55 & 1.96 & 78 & 84 & 83 & 95 & 47 & 347 \\
\hline 386.24 & D & 48 & & & & & & & & & & & & & & & \\
\hline 387.04 & $\mathrm{D}$ & 35 & 79.38 & 0.35 & 6.15 & 0.05 & 2.94 & 1.90 & 0.07 & 0.84 & 1.30 & 58 & 54 & 46 & 85 & 46 & 266 \\
\hline 388.04 & $\mathrm{D}$ & 53 & 80.03 & 0.33 & 5.90 & 0.05 & 2.84 & 180 & 0.07 & 0.70 & 124 & 55 & 56 & 47 & 87 & 48 & 288 \\
\hline 388.56 & D & 52 & 78.87 & 0.38 & 6.35 & 0.06 & 2.93 & 1.84 & 0.07 & 0.95 & 1.34 & 59 & 66 & 41 & 101 & 53 & 306 \\
\hline 390.04 & $\mathrm{D}$ & 51 & & & & & & & & & & & & & & & \\
\hline 391.24 & $\mathrm{D}$ & 40 & 78.68 & 0.47 & 6.24 & 0.12 & 2.99 & 1.90 & 0.07 & 0.95 & 1.22 & 64 & 60 & 50 & 155 & 75 & 324 \\
\hline 391.94 & $\mathrm{D}$ & 58 & & & & & & & & & & & & & & & \\
\hline 392.94 & $\mathrm{D}$ & 51 & 82.15 & 0.26 & 4.70 & 0.03 & 2.34 & 1.56 & 0.06 & 0.46 & 0.96 & 45 & 45 & 47 & 82 & 46 & 225 \\
\hline 394.06 & $\mathrm{D}$ & 70 & & & & & & & & & & & & & & & \\
\hline 394.94 & $\mathrm{D}$ & 64 & 81.28 & 0.28 & 4.95 & 0.05 & 2.50 & 1.66 & 0.08 & 0.50 & 0.97 & 58 & 77 & 48 & 54 & 55 & 215 \\
\hline 395.94 & $\mathrm{D}$ & 64 & & & & & & & & & & & & & & & \\
\hline 397.34 & D & 57 & & & & & & & & & & & & & & & \\
\hline 398.24 & $\mathrm{D}$ & 37 & & & & & & & & & & & & & & & \\
\hline 399.04 & $\mathrm{D}$ & 33 & 80.43 & 0.29 & 5.17 & 0.04 & 2.57 & 1.68 & 0.07 & 0.58 & 1.08 & 49 & 51 & 44 & 90 & 47 & 233 \\
\hline 400.34 & D & 56 & & & & & & & & & & & & & & & \\
\hline 401.34 & $\mathrm{D}$ & 38 & 74.63 & 0.49 & 7.50 & 0.10 & 3.40 & 2.37 & 0.08 & 0.93 & 1.44 & 84 & 96 & 51 & 120 & 98 & 358 \\
\hline 401.84 & $\mathrm{D}$ & 43 & & & & & & & & & & & & & & & \\
\hline 403.24 & D & 45 & 80.09 & 0.51 & 5.43 & 0.09 & 3.03 & 1.82 & 0.08 & 1.07 & 1.01 & 84 & 96 & 51 & 120 & 98 & 358 \\
\hline 404.04 & $\mathrm{D}$ & 46 & 81.58 & 0.57 & 4.45 & 0.10 & 2.66 & 1.56 & 0.07 & 1.54 & 0.79 & 66 & 40 & 31 & 235 & 107 & 333 \\
\hline 405.04 & $\mathrm{D}$ & 57 & & & & & & & & & & & & & & & \\
\hline 405.88 & $\mathrm{D}$ & 37 & 66.00 & 0.40 & 4.22 & 0.09 & 11.23 & 6.65 & 0.18 & 2.47 & 0.69 & 100 & 167 & 24 & 175 & 71 & 118 \\
\hline 407.44 & $\mathrm{D}$ & 39 & 79.48 & 0.40 & 4.12 & 0.08 & 3.03 & 1.92 & 0.08 & 0.91 & 0.73 & 71 & 93 & 32 & 99 & 78 & 163 \\
\hline 408.04 & $\mathrm{D}$ & 65 & 85.06 & 0.37 & 3.32 & 0.06 & 1.89 & 1.10 & 0.07 & 0.76 & 0.62 & 57 & 73 & 26 & 84 & 74 & 369 \\
\hline 409.04 & $\mathrm{D}$ & 52 & & & & & & & & & & & & & & & \\
\hline 409.94 & $\mathrm{D}$ & 46 & 83.06 & 0.41 & 2.97 & 0.07 & 2.38 & 1.31 & 0.08 & 1.01 & 0.51 & 63 & 64 & 17 & 87 & 67 & 272 \\
\hline 411.84 & $\mathrm{D}$ & 30 & 76.63 & 0.96 & 6.12 & 0.18 & 3.91 & 2.11 & 0.11 & 2.66 & 0.92 & 106 & 81 & 34 & 264 & 207 & 289 \\
\hline 412.24 & $\mathrm{D}$ & 30 & 82.68 & 0.53 & 4.10 & 0.09 & 2.34 & 1.36 & 0.08 & 1.21 & 0.70 & 69 & 56 & 28 & 125 & 115 & 182 \\
\hline 413.04 & SA & 33 & 69.67 & 0.86 & 9.40 & 0.19 & 4.62 & 2.36 & 0.13 & 2.62 & 1.35 & 98 & 88 & 39 & 291 & 372 & 277 \\
\hline 414.04 & $\mathrm{D}$ & 48 & 79.24 & 0.74 & 5.14 & 0.15 & 3.22 & 1.84 & 0.09 & 1.95 & 0.80 & 87 & 111 & 32 & 210 & 151 & 291 \\
\hline 415.16 & $\mathrm{D}$ & 39 & & & & & & & & & & & & & & & \\
\hline 415.94 & $\mathrm{D}$ & 36 & 81.03 & 0.54 & 4.08 & 0.13 & 2.93 & 1.76 & 0.09 & 1.42 & 0.66 & 74 & 82 & 28 & 157 & 114 & 202 \\
\hline 417.04 & D & 64 & 80.20 & 0.60 & 4.89 & 0.13 & 3.05 & 1.65 & 0.08 & 1.35 & 0.82 & 83 & 68 & 36 & 159 & 127 & 214 \\
\hline 418.15 & $\mathrm{D}$ & 63 & 81.55 & 0.35 & 5.16 & 0.06 & 2.24 & 1.47 & 0.07 & 0.57 & 1.00 & 82 & 80 & 44 & 68 & 70 & 196 \\
\hline 419.14 & $\mathrm{D}$ & 46 & & & & & & & & & & & & & & & \\
\hline 420.36 & $\mathrm{D}$ & 43 & 8121 & 0.38 & 3.97 & 0.07 & 2.48 & 152 & 0.08 & 0.74 & 0.70 & 68 & 75 & 29 & 95 & 69 & 833 \\
\hline 421.16 & $\mathrm{D}$ & 54 & 78.92 & 0.48 & 4.88 & 0.10 & 3.04 & 1.93 & 0.08 & 0.93 & 0.85 & 78 & 75 & 37 & 113 & 86 & 200 \\
\hline 421.74 & $\mathrm{D}$ & 41 & 78.10 & 0.53 & 4.53 & 0.10 & 3.21 & 1.98 & 0.08 & 1.16 & 0.74 & 82 & 67 & 31 & 140 & 101 & 185 \\
\hline 423.36 & $\mathrm{D}$ & 39 & & & & & & & & & & & & & & & \\
\hline 424.06 & $\mathrm{D}$ & 30 & & & & & & & & & & & & & & & \\
\hline 425.26 & $\mathrm{D}$ & 65 & 82.47 & 0.42 & 3.97 & 0.08 & 2.20 & 1.14 & 0.07 & 0.68 & 0.69 & 62 & 66 & 28 & 92 & 70 & 215 \\
\hline 425.60 & dol & 13 & 41.31 & 0.21 & 2.50 & 0.09 & 3.94 & 10.52 & 0.14 & 13.51 & 0.40 & 63 & 51 & 15 & 338 & 99 & 1237 \\
\hline 425.94 & $\mathrm{D}$ & 48 & & & & & & & & & & & & & & & \\
\hline 426.66 & dol & 9 & 36.49 & 0.15 & 2.04 & 0.11 & 2.97 & 11.62 & 0.17 & 15.90 & 0.33 & 49 & 35 & 13 & 546 & 62 & 239 \\
\hline 428.24 & $\mathrm{D}$ & 58 & 83.67 & 0.28 & 3.51 & 0.05 & 1.88 & 1.06 & 0.07 & 0.45 & 0.64 & 51 & 58 & 24 & 51 & 48 & 167 \\
\hline 429.76 & $\mathrm{D}$ & 68 & 84.55 & 0.26 & 3.30 & 0.04 & 1.89 & 1.07 & 0.07 & 0.41 & 0.64 & 47 & 61 & 31 & 63 & 52 & 178 \\
\hline 431.74 & $\mathrm{D}$ & 46 & 83.09 & 0.22 & 3.84 & 0.03 & 1.97 & 1.25 & 0.07 & 0.41 & 0.74 & 51 & 62 & 38 & 52 & 54 & 187 \\
\hline
\end{tabular}


Table 1 (continued)

\begin{tabular}{|c|c|c|c|c|c|c|c|c|c|c|c|c|c|c|c|c|c|}
\hline mbsf & Lithology & $\begin{array}{l}\text { Biog. opal } \\
\text { (wt.\%) }\end{array}$ & $\begin{array}{l}\text { Si02 } \\
\text { (wt.\%) }\end{array}$ & $\begin{array}{l}\text { Ti02 } \\
\text { (wt.\%) }\end{array}$ & $\begin{array}{l}\text { A1203 } \\
\text { (wt.\%) }\end{array}$ & $\begin{array}{l}\mathrm{P}_{2} \mathrm{O}_{5} \\
\text { (wt.\%) }\end{array}$ & $\begin{array}{l}\text { Fe203 } \\
\text { (wt.\%) }\end{array}$ & $\begin{array}{l}\mathrm{MgO} \\
\text { (wt.\%) }\end{array}$ & $\begin{array}{l}\text { Mno } \\
\text { (wt.\%) }\end{array}$ & $\begin{array}{l}\mathrm{CaO} \\
\text { (wt.\%) }\end{array}$ & $\begin{array}{l}\text { K20 } \\
\text { (wt.\%) }\end{array}$ & $\begin{array}{l}\text { V } \\
(\mathrm{ppm})\end{array}$ & $\begin{array}{l}\mathrm{Zn} \\
(\mathrm{ppm})\end{array}$ & $\begin{array}{l}\mathrm{Rb} \\
\mathrm{ppm}\end{array}$ & $\begin{array}{l}\mathrm{Sr} \\
(\mathrm{ppm})\end{array}$ & $\begin{array}{l}\mathrm{Zr} \\
(\mathrm{ppm})\end{array}$ & $\begin{array}{l}\mathrm{Ba} \\
(\mathrm{ppm})\end{array}$ \\
\hline 433.04 & $\mathrm{D}$ & 69 & 83.94 & 0.24 & 3.72 & 0.04 & 2.08 & 1.15 & 0.07 & 0.38 & 0.71 & 50 & 60 & 34 & 55 & 50 & 247 \\
\hline 434.46 & $\mathrm{D}$ & 64 & 84.17 & 0.27 & 3.41 & 0.05 & 1.99 & 1.08 & 0.07 & 0.43 & 0.65 & 50 & 57 & 30 & 66 & 56 & 204 \\
\hline 436.46 & $\mathrm{D}$ & 50 & 82.65 & 0.28 & 4.50 & 0.04 & 2.13 & 1.42 & 0.07 & 0.46 & 0.87 & 62 & 69 & 43 & 65 & 63 & 262 \\
\hline 437.10 & dol & 6 & 13.03 & 0.25 & 2.24 & 0.38 & 2.08 & 18.65 & 0.19 & 25.20 & 0.32 & 24 & 25 & 12 & 1079 & 74 & 726 \\
\hline 437.30 & $\mathrm{D}$ & 47 & & & & & & & & & & & & & & & \\
\hline 438.26 & $\mathrm{D}$ & 69 & 81.69 & 0.24 & 4.85 & 0.03 & 2.37 & 1.43 & 0.07 & 0.58 & 0.98 & 51 & 51 & 47 & 57 & 57 & 163 \\
\hline 438.94 & D & 78 & & & & & & & & & & & & & & & \\
\hline 440.32 & D & 47 & 83.44 & 0.45 & 3.58 & 0.07 & 1.84 & 1.06 & 0.07 & 0.67 & 0.64 & 83 & 87 & 27 & 100 & 87 & 176 \\
\hline 441.44 & D & 38 & & & & & & & & & & & & & & & \\
\hline 442.34 & D & 62 & 84.51 & 0.27 & 2.81 & 0.04 & 1.66 & 0.88 & 0.07 & 0.50 & 0.53 & 50 & 68 & 20 & 63 & 45 & 189 \\
\hline 443.24 & D & 47 & 83.77 & 0.29 & 3.55 & 0.04 & 1.79 & 1.07 & 0.07 & 0.54 & 0.67 & 63 & 63 & 30 & 63 & 51 & 155 \\
\hline 443.74 & D & 40 & 82.58 & 0.36 & 3.67 & 0.06 & 1.95 & 1.12 & 0.07 & 0.74 & 0.65 & 58 & 60 & 28 & 84 & 58 & 154 \\
\hline 445.04 & $\mathrm{D}$ & 40 & & & & & & & & & & & & & & & \\
\hline 445.94 & D & 53 & 83.18 & 0.37 & 3.01 & 0.07 & 1.88 & 0.84 & 0.07 & 0.61 & 0.51 & 60 & 61 & 15 & 68 & 46 & 172 \\
\hline 447.18 & D & 62 & & & & & & & & & & & & & & & \\
\hline 448.18 & $\mathrm{D}$ & 43 & 84.48 & 0.38 & 2.86 & 0.07 & 1.49 & 0.79 & 0.07 & 0.65 & 0.50 & 61 & 67 & 20 & 92 & 68 & 144 \\
\hline 449.26 & dol & 9 & 21.91 & 0.14 & 1.24 & 0.11 & 2.06 & 16.06 & 0.20 & 21.91 & 0.17 & 19 & 22 & 8 & 879 & 48 & 114 \\
\hline 450.16 & $\mathrm{D}$ & 37 & & & & & & & & & & & & & & & \\
\hline 451.24 & D & 43 & 78.44 & 0.49 & 7.00 & 0.07 & 2.76 & 1.73 & 0.07 & 0.70 & 1.32 & 106 & 81 & 65 & 106 & 90 & 256 \\
\hline 452.14 & D & 43 & 82.73 & 0.38 & 3.65 & 0.06 & 2.06 & 1.10 & 0.08 & 0.66 & 0.62 & 64 & 52 & 26 & 88 & 63 & 221 \\
\hline 453.14 & D & 34 & 77.68 & 0.62 & 4.27 & 0.15 & 3.63 & 1.66 & 0.12 & 1.81 & 0.63 & 91 & 75 & 25 & 178 & 105 & 208 \\
\hline 453.84 & $\mathrm{D}$ & 32 & 77.48 & 0.60 & 4.78 & 0.11 & 3.75 & 1.73 & 0.10 & 1.31 & 0.73 & 93 & 72 & 33 & 168 & 106 & 189 \\
\hline 455.44 & $\mathrm{D}$ & 70 & & & & & & & & & & & & & & & \\
\hline 456.14 & $\mathrm{D}$ & 52 & 81.67 & 0.32 & 3.25 & 0.05 & 2.88 & 1.24 & 0.09 & 0.71 & 0.57 & 65 & 86 & 24 & 90 & 61 & 158 \\
\hline 457.14 & D & 55 & 82.84 & 0.32 & 3.55 & 0.05 & 2.12 & 1.16 & 0.08 & 0.61 & 0.62 & 66 & 69 & 28 & 79 & 58 & 161 \\
\hline 458.44 & $\mathrm{D}$ & 37 & 82.42 & 0.32 & 2.85 & 0.06 & 2.40 & 1.19 & 0.08 & 0.68 & 0.49 & 57 & 62 & 21 & 90 & 61 & 117 \\
\hline 459.18 & $\mathrm{D}$ & 53 & & & & & & & & & & & & & & & \\
\hline 459.76 & SS & 10 & & & & & & & & & & & & & & & \\
\hline 460.76 & SS & 21 & 64.20 & 0.73 & 12.33 & 0.14 & 6.72 & 4.07 & 0.10 & 1.27 & 2.45 & 143 & 86 & 116 & 161 & 126 & 349 \\
\hline 462.26 & SS & 7 & 58.21 & 0.78 & 12.52 & 0.22 & 6.07 & 4.98 & 0.15 & 3.92 & 2.28 & 118 & 96 & 98 & 204 & 144 & 438 \\
\hline 463.14 & Dc & 8 & & & & & & & & & & & & & & & \\
\hline 464.34 & Dc & 15 & 58.75 & 0.81 & 12.79 & 0.20 & 6.17 & 5.01 & 0.14 & 3.67 & 2.38 & 127 & 96 & 104 & 208 & 152 & 403 \\
\hline 465.04 & Dc & 13 & & & & & & & & & & & & & & & \\
\hline 465.94 & Dc & 12 & & & & & & & & & & & & & & & \\
\hline 467.24 & Dc & 6 & 56.95 & 0.80 & 12.48 & 0.18 & 6.41 & 5.36 & 0.15 & 4.15 & 2.06 & 123 & 100 & 140 & 266 & 182 & 339 \\
\hline 467.94 & SS & 11 & 59.40 & 0.93 & 12.95 & 0.23 & 6.37 & 4.64 & 0.13 & 3.64 & 2.41 & 136 & 96 & 91 & 202 & 135 & 379 \\
\hline 469.14 & SS & 11 & 58.58 & 0.94 & 13.18 & 0.23 & 6.90 & 4.68 & 0.13 & 3.58 & 2.49 & 131 & 90 & 104 & 244 & 147 & 361 \\
\hline 470.34 & Dc & 8 & 52.17 & 1.51 & 11.78 & 0.39 & 7.92 & 4.87 & 0.25 & 9.18 & 1.78 & 170 & 91 & 74 & 445 & 220 & 350 \\
\hline 471.34 & Dc & 8 & & & & & & & & & & & & & & & \\
\hline 471.84 & SS & 12 & & & & & & & & & & & & & & & \\
\hline 473.02 & SS & 13 & 58.96 & 0.64 & 12.10 & 0.13 & 7.79 & 5.05 & 0.14 & 2.84 & 2.32 & 124 & 113 & 108 & 182 & 118 & 305 \\
\hline 474.14 & Dc & 7 & & & & & & & & & & & & & & & \\
\hline 474.94 & Dc & 9 & 60.18 & 1.07 & 12.71 & 0.25 & 6.92 & 4.73 & 0.14 & 3.98 & 2.31 & 140 & 100 & 97 & 292 & 190 & 374 \\
\hline 476.24 & Dc & 12 & & & & & & & & & & & & & & & \\
\hline 477.34 & Dc & 12 & 60.57 & 1.07 & 12.89 & 0.26 & 6.71 & 4.69 & 0.14 & 4.01 & 2.30 & 137 & 104 & 100 & 298 & 205 & 361 \\
\hline 478.34 & Dc & 9 & & & & & & & & & & & & & & & \\
\hline 479.44 & Dc & 18 & & & & & & & & & & & & & & & \\
\hline 480.14 & SS & 8 & 61.02 & 0.84 & 12.59 & 0.19 & 6.44 & 4.63 & 0.14 & 3.20 & 2.33 & 128 & 106 & 112 & 246 & 186 & 361 \\
\hline
\end{tabular}

\subsection{Element geochemistry}

Cluster analysis is a useful tool to rapidly obtain a perspective view on the geochemical behaviour of elements and their distribution in sediments. CA performed on samples (Fig. 2B) show that cluster S1 includes lithologies controlled by an increase in marine productivity (Bunt, 1973). In particular, sub-cluster S1a contains three samples at $426.66,437.10$ and 449.26 mbsf characterized by the presence of Fe-dolomite and sub-cluster S1b groups diatomite samples including samples from 363 to $380 \mathrm{mbsf}$ (in bold) recording the transition from open ocean to progressively more ice-proximal facies. Cluster S2 contains all other lithologies (diamictites, sandstones and siltstones/ shales).

To a first approximation, the dendrogram reported in Fig. 2A discriminates elements related to terrigenous material deriving from physic and chemical weathering of source rocks (cluster E1) and elements of biogenic origin including biogenic opal (cluster E2).

Elements in $\mathrm{E} 1$ are, in turn, separated in sub-clusters $\mathrm{E} 1 \mathrm{a}\left(\mathrm{Rb}, \mathrm{K}_{2} \mathrm{O}\right.$, and $\left.\mathrm{Al}_{2} \mathrm{O}_{3}\right)$ and $\mathrm{E} 1 \mathrm{~b}\left(\mathrm{Zn}, \mathrm{Zr}, \mathrm{Fe}_{2} \mathrm{O}_{3}, \mathrm{P}_{2} \mathrm{O}_{5}, \mathrm{~V}\right.$, and $\left.\mathrm{TiO}_{2}\right)$. Previous studies on the Ross Sea have used geochemical datasets from Antarctic sedimentary records to assess changes in sediment provenance with depth and relative implication for glaciological models during Late Cenozoic time (Krissek and Kyle, 1998; Bellanca et al., 2000; Pompilio et al., 2007). McKay et al. (2009) and Monien et al. (2012) ascribed the investigated portion of the AND-1B core to the "sedimentological motif 2" related to interglacial periods in which sediment composition is mainly controlled by southern TAM (Transantarctic Mountains south of Ross Island) sources. Recently, Konfirst et al. (2012) recognized, within the 80 m-thick diatom interval, geochemical variations driven by changes in depositional processes resulting in alternations of Ti-rich sediments associated with Ross Sea volcanic sedimentary sources (primarily from McMurdo Volcanic Group, MVG) and K-rich terrigenous TAMsourced sediments. CA results presented here discriminate $\mathrm{K}$ and $\mathrm{Ti}$ in different sub-clusters (E1a and E1b, respectively), support this interpretation. As stated above, biogenic opal concentrations and Chaetoceros spp. data give evidence of stratified surface waters and high primary productivity levels, which are recognized as 

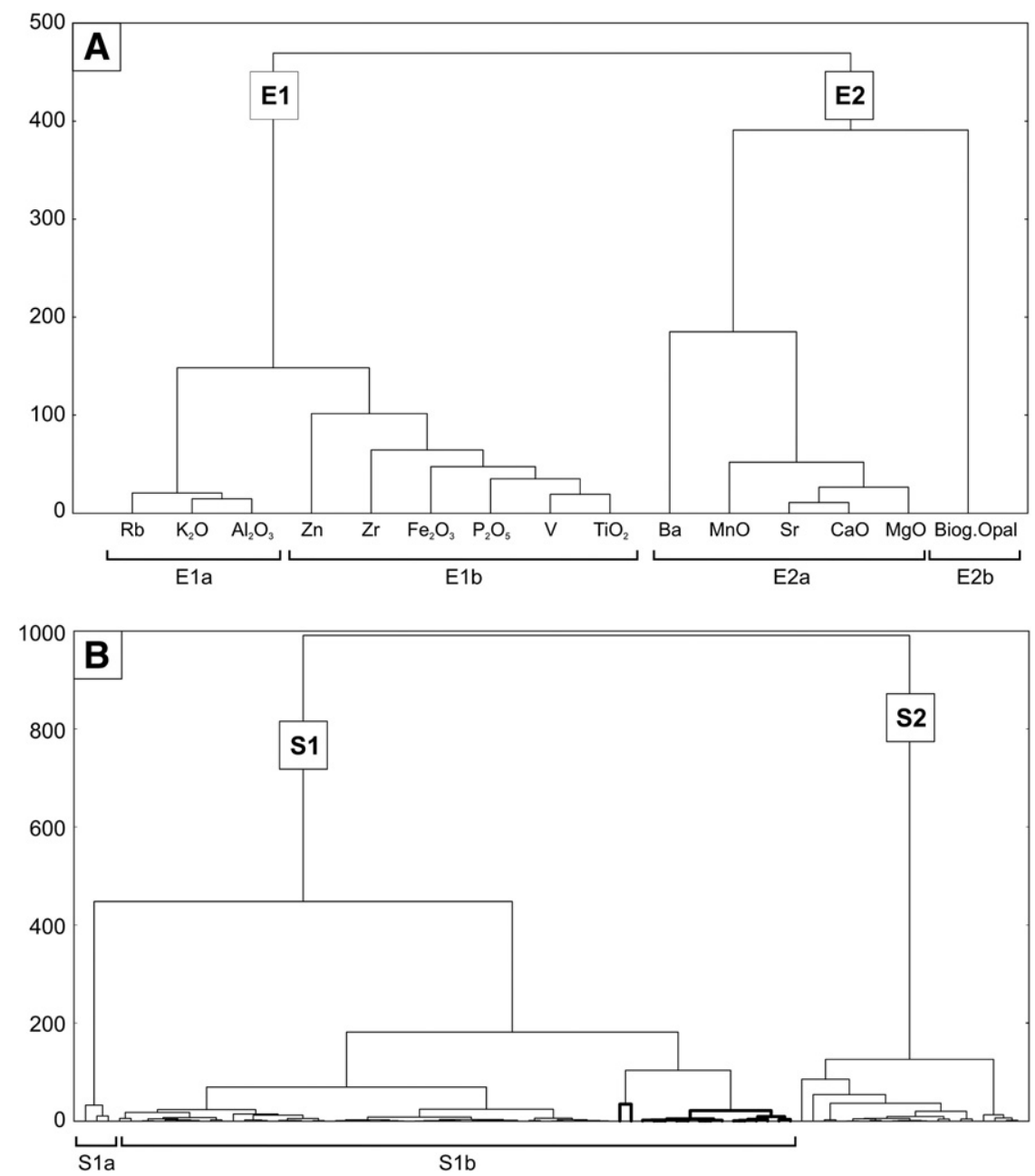

Fig. 2. Cluster analysis performed using the Ward's linkage method based on the Squared Euclidean distance on: (A) 16 variables and (B) 83 sampling sites.

factors triggering fluctuating anoxic-euxinic conditions at sea-sediment interface. In such a scenario, elemental signatures from this stratigraphic interval, which records warmer sea surface temperatures, little or no summer sea ice, and an open marine Ross embayment (Pollard and DeConto, 2009), could be influenced, as well as its source, by changes in depositional/early diagenetic conditions at the seafloor or over the first centimetres of sediment.

Element distribution profiles throughout the investigated AND-1B core portion show the strong control of biogenic silica dilution effect (Fig. 3). To avoid this effect, element concentrations were normalized to Al. Although removal of some $\mathrm{Al}$ amount from seawater by the oxide components cannot be ruled out (Kryc et al., 2003), this element is generally assumed to represent a reliable measure of the terrigenous load in marine sediments. Another reason for using $\mathrm{Al}$ is that this element is typically not diagenetically labile. Taking into account that Ti-rich sediments were related to MVG source (Konfirst et al., 2012; Monien et al., 2012), the relationships of Ti with other elements grouping in the same cluster E1b have been investigated by binary diagrams proposed in Fig. 5. The presence of an immobile element in the denominator of both ratios, allows to discriminate amongst elements with different mobility and/or involved in different depositional processes (Fralick and Kronberg, 1997). In calculating $r$ values, samples enriched in Fe-dolomite and three outliers (samples at 413,04 mbsf for $\mathrm{Zr}$ and at 405,88 mbsf for Fe and $\mathrm{Zn}$; Fig. 5) were discarded (see discussion below). P and $\mathrm{Zr}$ and, to a lesser extent, Fe are well correlated with $\mathrm{Ti}(\mathrm{r}=0.91,0.72$, and 0.66 respectivelly). Beyond than the provenance (see above),
Ti is often associated with dominant aeolian inputs (e.g. Boyle, 1983; Schimmield, 1992; Bertrand et al., 1996; Brumsack, 2006) and Pompilio et al. (2007) found that, for AND-1B core, Zr typically indicates volcanic material like ashes from surrounding volcanoes. Moreover, several recent studies have confirmed that a portion of Fe can be released from sea ice and icebergs playing a key role in triggering and regulating large-scale phytoplankton blooms when it is released to the water column (Sedwick and DiTullio, 1997; Sedwick et al., 2000; Lannuzel et al., 2007; Raiswell et al., 2008). Finally, P probably follows same fate of iron being adsorbed onto Fe-oxyhydroxides coatings (e.g. Jarvis et al., 1994; Piper and Perkins, 2004; Scopelliti et al., 2010). On the basis of these considerations, data presented here, suggest that the seafloor was influenced by aeolian particles that have settled through the water column, at the time of diatomite deposition. Consistently, sediment blown onto the sea ice and released to the water column during the melting has long been suggested as the dominant source of modern lithogenic sediment in McMurdo Sound (Bentley, 1979; Barrett et al., 1983; Macpherson, 1987; Atkins and Dunbar, 2009; Dunbar et al., 2009). Moreover, increases of Ti/Al ratios within two intervals at 400-425 and 440-460 mbsf (Fig. 6) correspond to decreases of Chaetoceros spp. abundances (Fig. 3) consisting with periods of ice-free, open water (see Section 5.1), which well fits with increases in particles released to the water column during the ice melting.

With respect to $\mathrm{V}$ and $\mathrm{Zn}$ vs. Ti a more scattered pattern (Fig. 5D and E), in particular for diatomite and Fe-dolomite samples is detectable, meaning that other processes must be invoked. After normalization to 

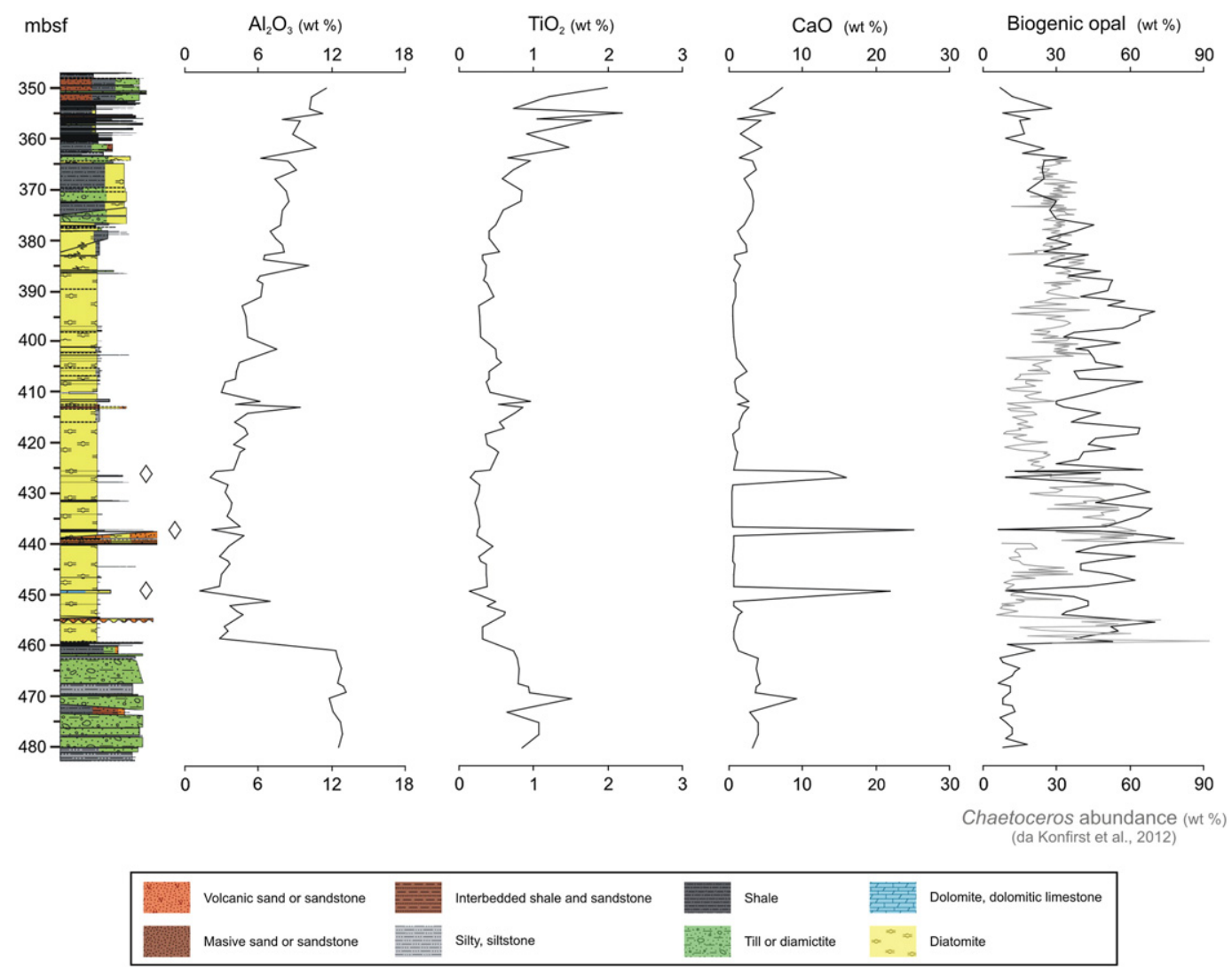

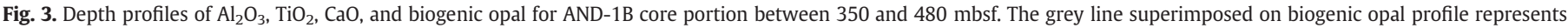
Chaetoceros spp. abundances from Konfirst et al. (2012). Lithostratigraphy from Naish et al. (2007). Rhombs indicate Fe-dolomite bearing samples.

Al, their depth profiles show (Fig. 6) a clear but fluctuating increase between 400 and 460 mbsf. In oxic water, vanadium is present as $\mathrm{V}(\mathrm{V})$ in form of vanadate oxyanions, and it is readily adsorbed onto both Mn- and Fe-oxyhydroxides (Calvert and Piper, 1984; Wehrly and Stumm, 1989) and possibly kaolinite (Breit and Wanty, 1991). Under weak reducing conditions, $\mathrm{V}(\mathrm{V})$ is reduced to $\mathrm{V}$ (IV), which may be removed to the sediment by surface adsorption processes or by formation of organometallic ligands (Emerson and Huested, 1991; Morford and Emerson, 1999). Under more strongly reducing/euxinic conditions, the presence of free $\mathrm{H}_{2} \mathrm{~S}$ released by bacterial sulphate reduction causes $\mathrm{V}$ to be further reduced to $\mathrm{V}$ (III), which can be taken up by geoporphyrins or be precipitated as oxide or hydroxide phase (Wanty and Goldhaber, 1992). Vanadium is thus probably not trapped in solid solution by Fe-sulphides (Algeo and Maynard, 2004) and may be removed from pore waters below the level of Mn-Fe oxyhydroxides reduction (Hastings et al., 1996; Morford and Emerson, 1999; Tribovillard et al., 2006). As Zinc, it exists, in oxic seawater, as soluble $\mathrm{Zn}^{2+}$ mostly in complexes with humic/fulvic acids (Calvert and Pedersen, 1993; Algeo and Maynard, 2004) or adsorbed onto particulate Fe-Mn oxyhydroxides (Fernex et al., 1992). During organic matter decay, Zn may be released from organometallic complexes to pore waters whilst under bacterial sulphate reduction may be trapped as sulphides (Jacobs et al., 1985). Greater enrichments in $\mathrm{Zn}$ with respect to Fe may be related to faster water exchange reaction kinetics of $\mathrm{Zn}^{2+}$ with respect to $\mathrm{Fe}^{2+}$, thus forming their own sulphide minerals (Morse and Luther, 1999; Scopelliti et al., 2006). Taking into account these considerations, increase in V and $\mathrm{Zn}$ in 400-460 mbsf interval within the diatomite interval testifies bio-accumulation and associated sulphidation processes persisting during the early diagenesis. Consistently, sulphide precipitation and incorporation of $\mathrm{Fe}^{2+}$ in pyrite can explain the fact that the correlation of Fe vs. Ti is significant $(r=0.66)$ but less pronounced than those between $\mathrm{Ti}$ and $\mathrm{P}, \mathrm{Zr}$. Sulphidation process probably culminates in the sample at 405,88 mbsf in which a significant enrichments in Fe and Zn (Fig. 5C and E) is documented probably owing to their trapping by sulphide precipitation.

To complete this picture, elements linked to biogenic origin and grouped in cluster E2 must be considered. $\mathrm{MnO}, \mathrm{Sr}, \mathrm{CaO}$, and $\mathrm{MgO}$ (sub-cluster E2a) are related to carbonate phases. Scopelliti et al. (2011) excluded that carbonates from the diatomite interval originated by late diagenetic processes and documented negative carbon isotope composition $\left(\delta^{13} \mathrm{C}\right.$ down to $-12.8 \%$ ) compatible with authigenic precipitation of carbonate in organic-rich sediments. In particular, the authors found persisting very negative signatures (mean value - $8.5 \%$ ) in 390-440 mbsf interval interpreted as indicative of high ${ }^{12} \mathrm{C}$ availability as a consequence of anoxic conditions and microbial oxidation of organic matter, which is consistent with sulphide precipitation (see discussion above).

As to barium, it was expected a strong relation with biogenic opal in accord with the hypothesis of Ba uptake by diatoms, inducing barite precipitation during their decay (Vinogradova and Koval'skiy, 1962; Bishop, 1988; Sternberg et al., 2005) but instead CA results highlight large Euclidean distance between biogenic opal and Ba for AND-1B samples investigated. Really, it is to consider the difficulty in laboratory experiments to well define the uptake of Ba by diatoms; moreover, the role of processes of precipitation/dissolution of barite and/or adsorption/desorption of $\mathrm{Ba}^{2+}$ on amorphous iron hydroxide in the oxygen minimum zone is still under debate. Nevertheless, the organic matter decay or the Ba desorption in the deeper water could lead to supersaturation and precipitation of barite, this being a more refractive species with respect to organic carbon, carbonate and opal. For this reason barium is often used as a primary productivity proxy (e.g., Dehairs et al., 1980, 1991; Dymond et al., 1992; François et al., 1995; McManus et al., 1998a,b, 1999; Ganeshram et al., 2003; Cardinal et al., 2005; Sanchez-Vidal et al., 2005; Henkel et al., 2012). In the AND-1B diatomite sequence, the $\mathrm{Ba} / \mathrm{Al}$ depth profile is characterized by few pronounced positive peaks, (i) at $~ 437$ mbsf just above 
the hiatus, (ii) at $\sim 420$ and $\sim 425 \mathrm{mbsf}$, and (iii) at $363 \mathrm{mbsf}$ at the top of diatomite interval (Fig. 6). Beside these peaks, Ba concentrations measured in the diatomite samples are lower (100-500 ppm; Table 1) than in other cores from the Antarctica margin showing Ba values that range between 600 and 2400 ppm (Bonn et al., 1998). An explanation for the measured low concentrations can be the oxy-hydroxide remobilization and sulphate reducing conditions producing a low degree of barite saturation in the first centimetres of sediment. Barite dissolution under anoxic conditions and consequent upward Ba diffusion into sulphate-bearing pore water lead to precipitation of authigenic barite in a diagenetic front. Thus, the barite front mainly marks the boundary between sulphate-rich and sulphate-poor pore waters (Cronan, 1974; Dean and Schreiber, 1978; von Breymann et al., 1992; Torres et al., 1996; Paytan et al., 2004; Hendy, 2010) that in the investigated AND-1B core portion can be identified with the Ba peak at the top of the diatomite sequence (at 363 mbsf). Strangely, other Ba peaks within the diatomite sequence are relative to samples in which the presence of pyrite and Fe-dolomite is documented (Fig. 7A). Actually, both these phases need sulphate reducing conditions for their precipitation because the reaction

$2 \mathrm{CH}_{2} \mathrm{O}+\mathrm{SO}_{4}^{2-} \rightarrow \mathrm{HCO}_{3}^{-}+\mathrm{HS}^{-}+\mathrm{CO}_{2}+\mathrm{H}_{2} \mathrm{O}$

reduces sulphate to sulphide for pyrite precipitation and increases $\mathrm{pH}$ and carbonate alkalinity for formation of Fe-dolomite and other authigenic carbonate (Vasconcelos and McKenzie, 1997; Warthmann et al., 2000; van Lith et al., 2003; Scopelliti et al., 2009). According to Riedinger et al. (2006), additional Ba peaks can be found below the
SMT as consequence of nonsteady-state processes characterized by increasing sedimentation rate or methane flux. Barium enrichments in sulphate-depleted conditions could be the consequence of (i) strikingly high amounts of total $\mathrm{Ba}$ in the sediment system influencing the dissolution rate of barite; (ii) occurrence of other Ba phases that show oxygen depletion relative to barite, such as those recognized by Gonzáles-Muñoz et al. (2003) consisting of Ba-containing spherical aggregates; (iii) or, as from laboratory results of Baldi et al. (1996), microbial reduction of barite via formation of other barium compounds, such as witherite $\left(\mathrm{BaCO}_{3}\right)$ and transient species of barium sulphide (BaS). Backscattered SEM image of the considered samples (Fig. 7B) highlights the coexistence of disseminated pyrite $(<10 \mu \mathrm{m}$ in size) with "brighter" crystals (white arrows), being the number of backscattered electrons (BSE) reaching the detector (and, as consequence, the brightness) proportional to the mean atomic number of the mineral. Chemical composition of these crystal is reported in EDS spectrum of Fig. 7C. For comparison, in Fig. 7D are reported the three EDS spectra from Riedinger et al. (2006) that show progressive oxygen decrease and carbon increase during the reduction of barium-sulphate leading to the formation of witherite via the transient phase barium sulphide:

$\mathrm{BaSO}_{4}+2 \mathrm{C} \rightarrow \mathrm{BaS}+2 \mathrm{CO}_{2}$

$\mathrm{BaS}+\mathrm{CO}_{2}+\mathrm{H}_{2} \mathrm{OBaCO}_{3}+\mathrm{H}_{2} \mathrm{~S}$

A short oxygen peak in AND-1B samples indicating the presence of an oxygen-depleted barium phase (Fig. 7C), which is reasonably

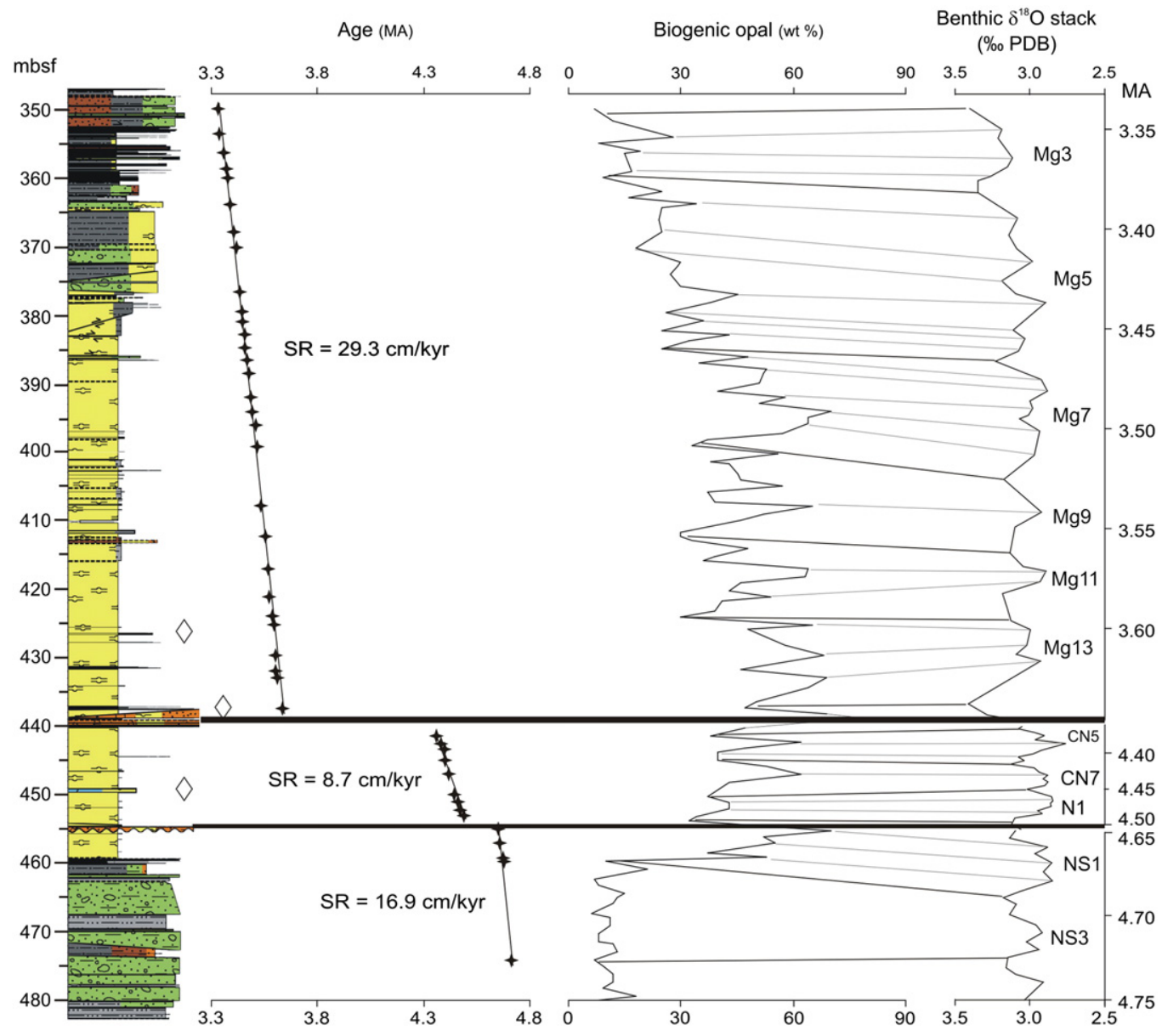

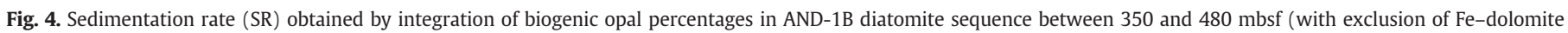
samples) with curve of benthic $\delta^{18}$ O LR04 stack (Lisiecki and Raymo, 2005). Lithologies and rhombs as in Fig. 3. 

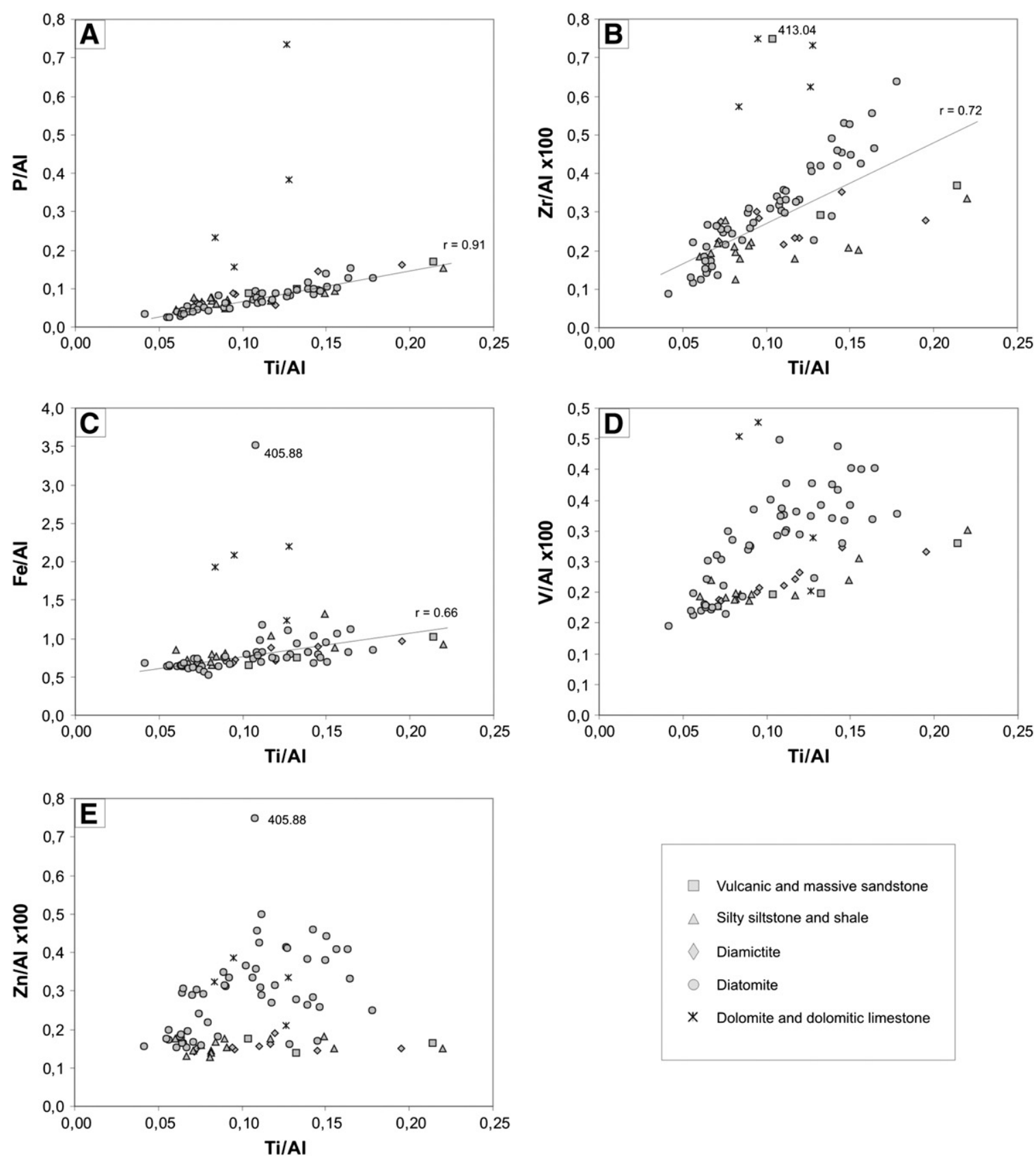

Fig. 5. Dispersion diagrams of $\mathrm{P} / \mathrm{Al}, \mathrm{Zr} / \mathrm{Al}, \mathrm{Fe} / \mathrm{Al}, \mathrm{V} / \mathrm{Al}$, and $\mathrm{Zn} / \mathrm{Al}$ vs. Ti/Al for AND-1B core portion between 350 and $480 \mathrm{mbsf}$.

related to formation of barium sulphide resulting from same process invoked by Riedinger et al. (2006). As to factors triggering these processes, variations in sediment composition, in depositional dynamics, or in the upward methane flux can be invoked (Riedinger et al., 2006). An explanation for the Ba-peak at 437.10 mbsf immediately above the hiatus (see Fig. 4) could be that changes in sedimentation rate may induce non-steady state diagenetic processes and can lead to localize accumulations of authigenic phases (Riedinger et al., 2006; Arndt et al., 2009; März et al., 2011). As to Ba peaks at 425.60 and $420.36 \mathrm{mbsf}$, they correspond to the decrease of Chaetoceros spp. abundance (see Fig. 3) which is ascribed to passage from stratified surface waters trapping nutrients to ice-free, more open water which could be considered as co-responsible in change of sedimentological system.

\section{Conclusions}

Results from the biogenic opal and element geochemistry study of the 350-480 mbsf AND-1B core portion highlight that the distribution of major and minor elements throughout the diatomite interval accounts for a terrigenous/biogenic control influencing the conditions at the sea floor.

Across the diatomite interval recording the early Pliocene warming event, the content in biogenic opal shows distinct fluctuations opposed to those recorded by the $\delta^{18} \mathrm{O}$ values testifying glacial fluctuation during the warming event. The correlation with obliquity cycles was used to better define the sedimentation rate throughout the sequence highlighting a drastic change in sedimentation rate at 440 mbsf. Comparison with Chaetoceros spp. abundance gives evidence of alternating 

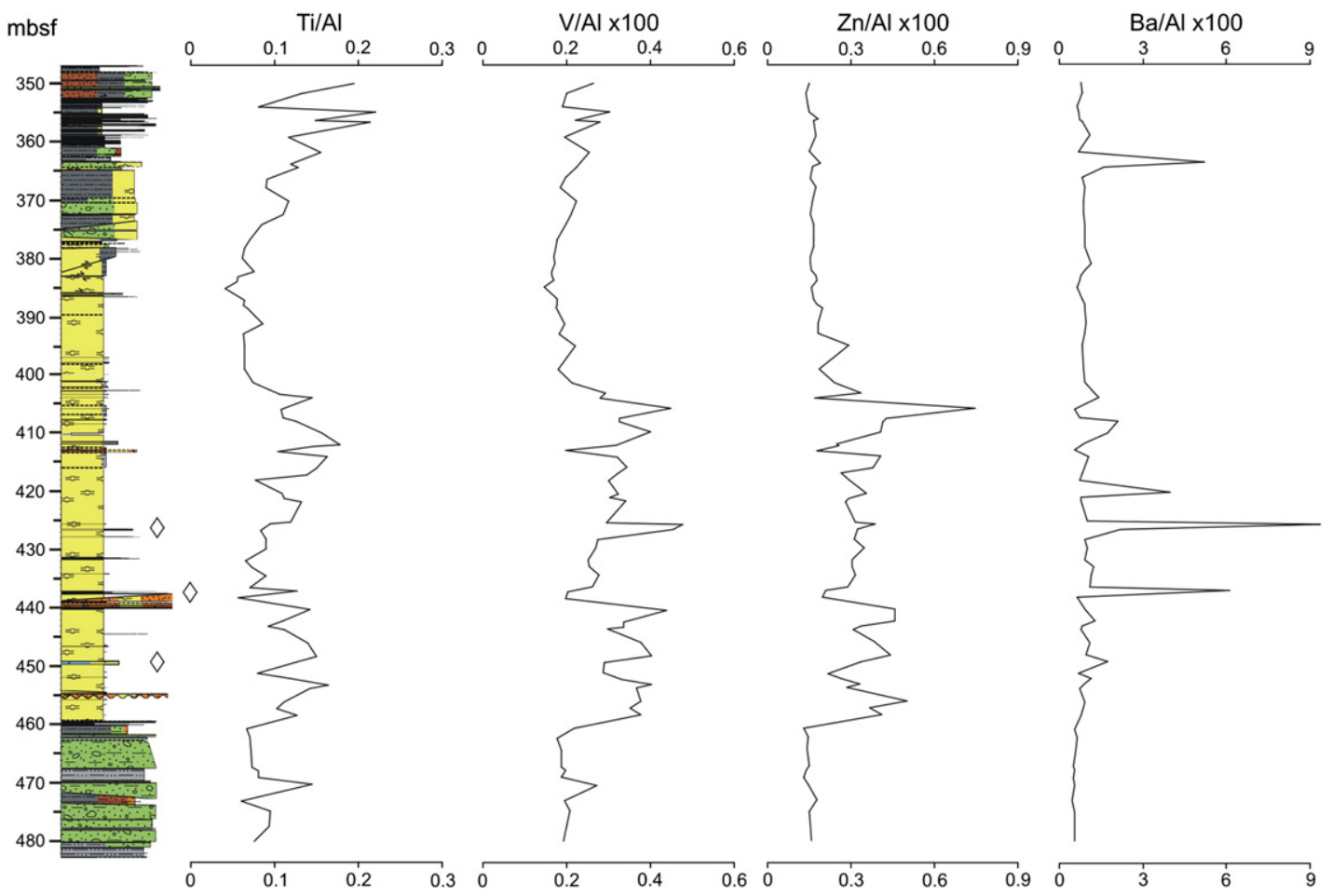

Fig. 6. Depth profiles of $\mathrm{Ti} / \mathrm{Al}, \mathrm{V} / \mathrm{Al}, \mathrm{Zn} / \mathrm{Al}$, and $\mathrm{Ba} / \mathrm{Al}$ for AND-1B core portion between 350 and $480 \mathrm{mbsf}$. Lithologies and rhombs as in Fig. 3 .
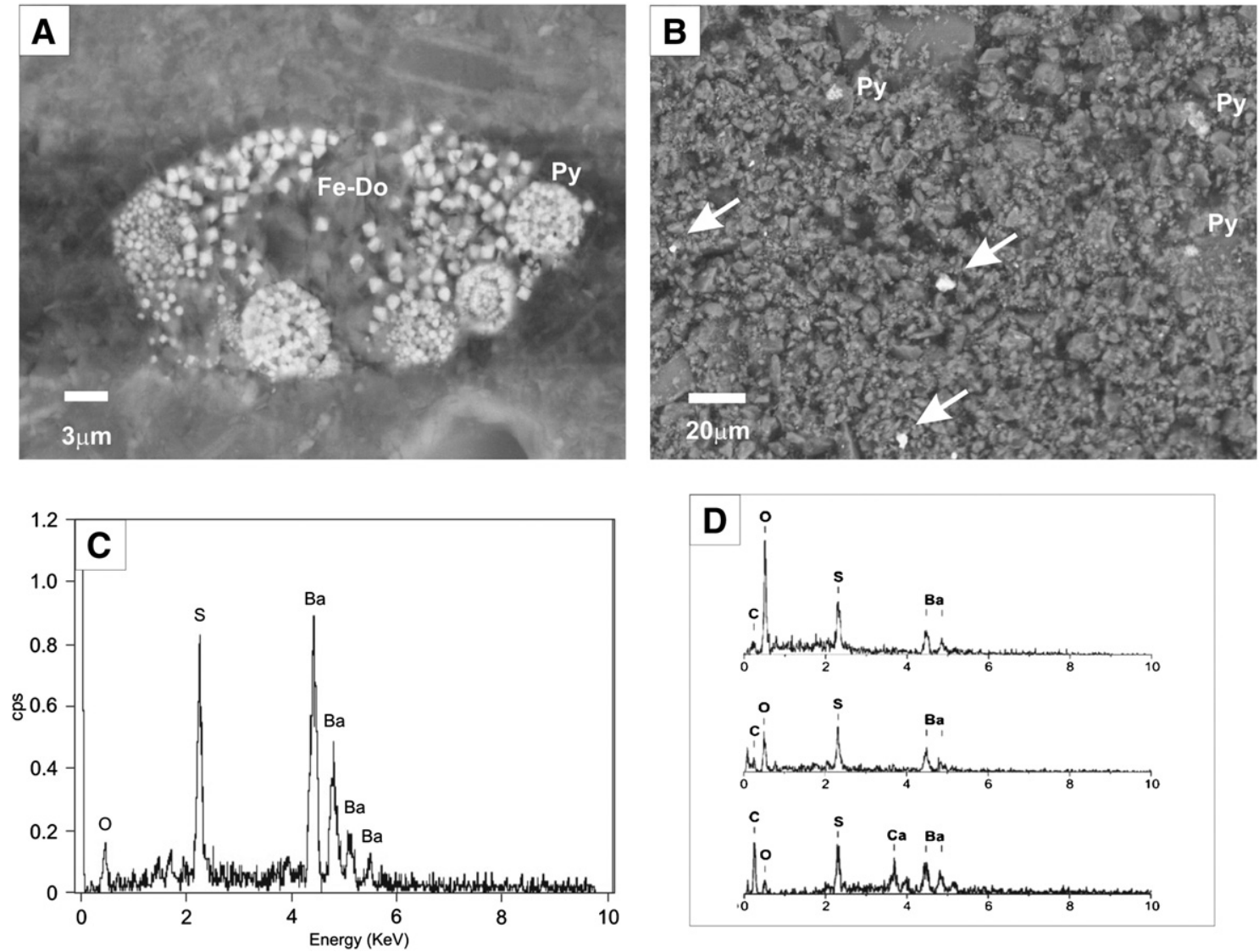

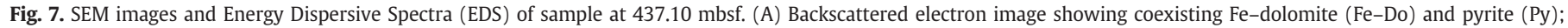

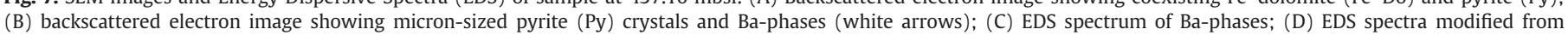
Riedinger et al. (2006) showing oxygen decrease from barite to witherite via barium sulphide (see text). 
conditions of stratified surface waters and terrigenous input triggering change in depositional/early diagenetic conditions at the sedimentwater interface. In this scenario, elements as $\mathrm{K}$ and Ti response to changes in terrigenous provenance and elements as V, Zn, Fe testify the presence of fluctuating reducing/anoxic conditions. Presence of barium enrichments in a such depositional environment in which this element undergoes remobilization is justified by variations in depositional dynmic induced by a drastic change in sedimentation rate and/or passage from surface waters characterized by high primary productivity to ice-free more open waters.

\section{Acknowledgements}

This study was supported by the Italian Antarctic Research (PNRA). ANDRILL-MIS Science Team, the Chief Scientists Tim Naish and Ross Powell are thanked for their encouragement and assistance. The ANDRILL project is a multinational collaboration between the Antarctic programmes of Germany, Italy, New Zealandand the United States. Antarctica New Zealand is the project operator and developed the drilling system in collaboration with Alex Pyne at Victoria University of Wellington and Webster Drilling and Enterprises Ltd. Antarctica New Zealand supported the drilling team at Scott Base; Raytheon Polar Services Corporation supported the science team at McMurdo Station and the Crary Science and Engineering Laboratory. The ANDRILL Science Management Office at the Universityof NebraskaLincoln provided science planning and operational support. Scientific studies have been jointly supported by the US National Science Foundation, NZ Foundation for Research Science and Technology, the Royal Society of NZ Marsden Fund, the Italian Antarctic Research Programme, and the German Research Foundation (DFG, KU 683/8) and the Alfred Wegener Institute Polar and Marine Research. The authors are indebted to two anonymous reviewers for their constructive comments that greatly improved the manuscript.

\section{References}

Algeo, T.J., Maynard, J.B., 2004. Trace-element behavior and redox facies in core shales of Upper Pennsylvanian Kansas-type cyclothems. Chemical Geology 206, 289-318.

Arndt, S., Hetzel, A., Brumsack, H.-J., 2009. Evolution of organic matter degradation in Cretaceous black shales inferred from authigenic barite: a reaction-transport model. Geochimica et Cosmochimica Acta 73, 2000-2022.

Atkins, C.B., Dunbar, G.B., 2009. Aeolian sediment flux from sea ice into Southern McMurdo Sound, Antarctica. Global and Planetary Change 69, 133-141.

Baldi, F., Pepi, M., Burrini, D., Kniewald, G., Scali, D., Lanciotti, E., 1996. Dissolution of barium from barite in sewage sludges and cultures of Desulfovibrio desulfuricans. Applied and Environmental Microbiology 62, 2398-2404.

Barrett, P.J., Pyne, A.R., Ward, B.L., 1983. Modern sedimentation in McMurdo Sound, Antarctica. In: Oliver, R.L., James, P.R., Jago, J.B. (Eds.), Antarctic Earth Science. Australian Academy of Sciences, Canberra, pp. 550-554.

Barron, J.A., 1992. Pliocene paleoclimatic interpretation of DSDP Site 580 (NW Pacific) using diatoms. Marine Micropaleontology 20, 23-44.

Bellanca, A., Neri, R., Sprovieri, M., 2000. Bulk geochemistry of the sand fraction from CRP-2/2A, Victoria Land Basin, Antartica. Terra Antartica 7, 553-560.

Bentley, P.N., 1979. Characteristics and distribution of wind blown sediment, Western McMurdo Sound, Antarctica. B.Sc. Hons Thesis, Victoria University of Wellington.

Bertrand, P., Shimmield, G., Martinez, P., Grousset, F., Jorissen, F., Paterne, M., Pujol, C., Bouloubassi, I., Buat Menard, P., Peypouquet, J.P., Beaufort, L., Sicre, M.A., LallierVerges, E., Foster, J.M., Ternois, Y., 1996. The glacial ocean productivity hypothesis: the importance of regional temporal and spatial studies. Marine Geology 130, 1-9.

Bishop, J.K.B., 1988. The barite-opal-organic carbon association in oceanic particulate matter. Nature 332, 341-343.

Bonn, W.J., Gingele, F.X., Grobe, H., Mackensen, A., Futterer, D.K., 1998. Palaeoproductivity at the Antartic continental margin: opal and barium records for the last 400 Ka. Palaeogeography, Palaeoclimatology, Palaeoecology 139, 195-211.

Boyle, E.A., 1983. Chemical accumulation variation under the Peru current during the last 130,000 years. Journal of Geophysical Research 88, 7667-7680.

Breit, G.N., Wanty, R.B., 1991. Vanadium accumulation in carbonaceous rocks: a review of geochemical controls during deposition and diagenesis. Chemical Geology 91, 83-97.

Brumsack, H.-J., 2006. The trace metal content of recent organic carbon-rich sediments: Implications for Cretaceous black shale formation. Palaeogeography, Palaeoclimatology, Palaeoecology 232 (2006), 344-361.

Brumsack, H.-J., Gieskes, J.M., 1983. Interstitial water trace-metal chemistry of laminated sediments from the Gulf of California, Mexico. Marine Chemistry 14, 89-106.
Bunt, J.S., 1973. Primary production: marine ecosystems. Human Ecology 1 (4), 333-345.

Calvert, S.E., Pedersen, T.F., 1993. Geochemistry of recent oxic and anoxic sediments: implications for the geological record. Marine Geology 113, 67-88.

Calvert, S.E., Piper, D.Z., 1984. Geochemistry of ferromanganese nodules: multiple diagenetic metal sources in the deep sea. Geochimica et Cosmochimica Acta 48, 1913-1928.

Cardinal, D., Savoye, N., Trull, T.W., André, L., Kopczynska, E.E., Dehairs, F., 2005. Variations of carbon remineralisation in the Southern Ocean illustrated by the Baxs proxy. Deep-Sea Research Part I: Oceanogrraphic Research Papers 52 355-370.

Castellini, D.G., Dickens, G.R., Snyder, G.T., Ruppel, C.D., 2006. Barium cycling in shallow sediment above active mud volcanoes in the Gulf of Mexico. Chemical Geology 226, 1-30.

Cronan, D.S., 1974. Authigenic minerals in deep-sea sediments. In: Goldberg, E.D. (Ed.) The Sea, 5. Wiley Interscience, pp. 491-525.

Cronin, T.M., Whatley, R., Wood, A., Tsukagoshi, A., Ikeya, N., Brouwers, E.M., Briggs Jr. W., 1993. Evidence for elevated mid-Pliocene temperatures in the Arctic Ocean based on marine ostracoda. Paleoceanography 8, 161-173.

Dean, W.E., Schreiber, B.C., 1978. Authigenic barite, leg 41 deep sea drilling project. In: Lancelot, Y., Seibold, E., et al. (Eds.), Proceedings of ODP, Initial Reports, 41. U.S Gov. Off., Washington, D.C., pp. 915-931.

Dehairs, F., Chesselet, R., Jedwab, J., 1980. Discrete suspended particles of barite and the barium cycle in the open ocean. Earth and Planetary Science Letters 49, 915-931.

Dehairs, F., Stroobants, N., Goeyens, L., 1991. Suspended barite as a tracer of biological activity in the Southern Ocean. Marine Chemistry 35, 399-410.

Dowsett, H., Barron, J., Poore, R., 1996. Middle Pliocene sea surface temperatures: a global reconstruction. Marine Micropaleontology 27, 13-25.

Dowsett, H.J., Chandler, M.A., Cronin, T.M., Dwyer, G.S., 2005. Middle Pliocene sea surface temperature variability. Paleoceanography 20, PA2014. http://dx.doi.org/ 10.1029/2005PA001133 (8 pp.).

Dunbar, G.B., Bertler, N.A.N., McKay, R.M., 2009. Sediment flux through the McMurdo Ice Shelf in Windless Bight, Antarctica. Global and Planetary Change 69, 87-93.

Dymond, J., Suess, E., Lyle, M., 1992. Barium in deep-sea sediments: a geochemical proxy for paleoproductivity. Paleoceanography 7, 163-181.

Emerson, S.R., Huested, S.S., 1991. Ocean anoxia and the concentration of molybdenum and vanadium in seawater. Marine Chemistry 34, 177-196.

Escutia, C., Bárcena, M.A., Lucchi, R.G., Romero, O., Ballegeer, A.M., Gonzalez, J.J., Harwood, D.M., 2009. Circum-Antarctic warming events between 4 and $3.5 \mathrm{Ma}$ recorded in marine sediments from the Prydz Bay (ODP Leg 188) and the Antarctic Peninsula (ODP Leg 178) margins. Global and Planetary Change 69, 170-184.

Fernex, F., Février, G., Benaïm, J., Arnoux, A., 1992. Copper, lead and zinc trapping in Mediterranean deep-sea sediments: probable coprecipitation with manganese and iron. Chemical Geology 98, 293-308.

Fielding, C., Whittaker, J., Henrys, S.A., Wilson, T.J., Naish, T.R., 2008. Seismic facies and stratigraphy of the Cenozoic succession in McMurdo Sound, Anatrctica: Implications for tectonic, climatic and glacial history. Palaeogeography, Palaeoclimatology, Palaeoecology 260, 8-29.

Fralick, P.W., Kronberg, B.I., 1997. Geochemical discrimination of clastic sedimentary rock sources. Sedimentary Geology 113, 111-124.

François, R., Honjo, S., Manganini, S.J., Ravizza, G.E., 1995. Biogenic barium fluxes to the deep sea: Implications for paleoproductivity reconstruction. Global Biogeochemical Cycles 9, 289-303.

Franzini, M., Leoni, L., Saitta, M., 1975. Revisione di una metodologia analitica per fluorescenza X basata sulla correzione completa degli effetti di matrice. Rendiconti Società Italiana Mineralogia e Petrologia 21, 99-108.

Ganeshram, R.S., Francois, R., Commeau, J., Brown-Leger, S.L., 2003. An experimental investigation of barite formation in seawater. Geochimica et Cosmochimica Acta 67 (14), 2599-2605.

Gingele, F., Dahmke, A., 1994. Discrete barite particles and barium as tracers of paleoproductivity in South Atlantic sediments. Paleoceanography 9, 151-168.

Gingele, F.X., Zabel, M., Kasten, S., Bonn, W.J., Nürnberg, C.C., 1999. Biogenic barium as a proxy for paleoproductivity: methods and limitations of application. In: Fischer, G. Wefer, G. (Eds.), Use of Proxies in Paleoceanography: Examples from the South Atlantic. Springer, pp. 345-364.

Giorgetti, G., Talarico, F., Sandroni, S., Zeoli, A., 2009. Provenance of Pleistocene sediments in the ANDRILL AND-1B drill core: clay and heavy mineral data. Global and Planetary Change 69, 94-102.

Gonzáles-Muñoz, M.T., Fernández-Luque, B., Martínez-Ruiz, F., Cheroun, K.B., Arias J.M., Rodríguez-Gallego, M., Martínez-Cañamero, M., de Linares, C., Paytan, A. 2003. Precipitation of barite by Myxococcus xanthus: possible implications for the biogeochemical cycle of barium. Applied and Environmental Microbiology 69, 5722-5725.

Grützner, J., Hillenbrand, C.-D., Rebesco, M., 2005. Terrigenous flux and biogenic silica deposition at the Antarctic continental rise during the late Miocene to early Pliocene: implications for ice sheet stability and sea icea coverage. Global and Planetary Change 45, 131-149.

Hastings, D.W., Emerson, S.R., Mix, A.C., 1996. Vanadium in foraminiferal calcite as a tracer for changes in the areal extent of reducing sediments. Paleoceanography $11,665-678$.

Haywood, A.M., Valdes, P.J., 2004. Modelling Pliocene warmth: contribution of atmosphere, oceans and cryospher. Earth and Planetary Science Letters 218, 363-377.

Hendy, I.L., 2010. Diagenetic behavior of barite in a coastal upwelling setting. Paleoceanography 25, PA4103. http://dx.doi.org/10.1029/2009PA001890.

Henkel, S., Mogollón, J.M., Nöthen, K., Franke, C., Bogus, K., Robin, E., Bahr, A. Blumenberg, M., Pape, T., Seifert, R., März, C., de Lange, G.J., Kasten, S., 2012. 
Diagenetic barium cycling in Black Sea sediments - a case study for anoxic marine environments. Geochimica et Cosmochimica Acta 88, 88-105. http://dx.doi.org/ 10.1016/j.gca.2012.04.021

Hepp, D.A., Mörz, T., Grützner, J., 2006. Pliocene glacial cyclicity in a deep-sea sediments drift (Antarctic Peninsula Pacific Margin). Palaeogeography, Palaeoclimatology, Palaeoecology 231, 181-198.

Hillenbrand, C.-D., Ehrmann, W., 2005. Late Neogene to Quaternary environmental changes in the Antarctic Peninsula region: evidence from drift sediments. Global and Planetary Change 45, 165-191.

Horgan, H., Naish, T.R., Bannister, S., Balfour, N., Wilson, G., 2005. Seismic stratigraphy of the Ross Island flexural moat under the McMurdo-Ross Ice Shelf, Antarctica, and a prognosis for stratigraphic drilling. Global and Planetary Change 45, 83-90.

Jacobs, L., Emerson, S.R., Skei, J., 1985. Partitioning and transport of metals across the $\mathrm{O}_{2} / \mathrm{H}_{2} \mathrm{~S}$ interface in a permanently anoxic basin, Framvaren Fjord, Norway. Geochimica et Cosmochimica Acta 49, 1433-1444.

Jarvis, I., Burnett, W.C., Nathan, Y., Almbaydin, F.S.M., Attia, A.K.M., Castro, L.N., Flicoteaux, R., Hilmy, M.E., Husain, V., Qutawnnah, A.A., Serjani, A., Zanin, Y.N. 1994. Phosphorite geochemistry: state of the art and environmental concerns. Eclogae Geologicae Helvetiae 87, 643-700.

Konfirst, M.A., Kuhn, G., Monien, D., Scherer, R.P., 2011. Correlation of Early Pliocene diatomite to low amplitude Milankovitch cycles in the ANDRILL AND-1B drill core. Marine Micropaleontology 80, 114-124.

Konfirst, M.A., Kuhn, G., Monien, D., Scherer, R.P., 2012. The influence of siliciclastic input on Chaetoceros abundance in an early Pliocene segment of the ANDRILL AND-1B drill core. Palaeogeography, Palaeoclimatology, Palaeoecology 346-347, 87-94.

Krissek, L.A., Kyle, P.R., 1998. Geochemical indicators of weathering and cenozoic palaeoclimates in sediments from CRP-1 and CIROS-1, McMurdo-1 Sound, Antartica. Terra Antarctica 5, 673-680.

Krissek, L.A., Browne, G., Carter, L., Cowan, E., Dunbar, G., McKay, R., Naish, T., Powell, R. Reed, J., Wilch, T., the ANDRILL-MIS Science Team, 2007. Sedimentology and stratigraphy of the ANDRILL McMurdo Ice Shelf (AND-1B) core: U.S. Geological Survey and The National Academies, Extended Abstract, 148 (USGS OF-20071047).

Kryc, K.A., Murray, R.W., Murray, D.W., 2003. Al-to-oxide and Ti-to-organic linkages in biogenic sediment: relationships to paleo-export production and bulk $\mathrm{Al} / \mathrm{Ti}$. Earth and Planetary Science Letters 211 (1-2), 125-141. http://dx.doi.org/10.1016/ S0012-821X(03)00136-5.

Lannuzel, D., Schoemann, V., de Jong, J.T.M., Tison, J.L., Chou, L., 2007. Distribution and biogeochemical behaviour of iron in the East Antarctic sea ice. Marine Chemistry $106,18-32$.

Leventer, A., Domack, E., Dunbar, R., Pike, J., Stickley, C., Maddison, E., Brachfeld, S. Manley, P., McClennen, C., 2006. Marine sediment record from the East Antarctic margin reveals dynamics of ice sheet recession. GSA Today $16,4-10$.

Lisiecki, L.E., Raymo, M.E., 2005. A Pliocene-Pleistocene stack of 57 globally distributed benthic d180 records. Paleoceanography 20, PA1003. http://dx.doi.org/10.1029/ 2004PA001071.

Macpherson, A.J., 1987. The MacKay Glacier/Granite Harbour system (Ross Dependency, Antarctica). A study in nearshore glacial marine sedimentation. Thesis. Victoria Univ. of Wellington, New Zealand. 85 pp.

Maddison, E.J., Pike, J., Leventer, A., Domack, E.W., 2005. Deglacial seasonal and subseasonal diatom record from Palmer Deep, Antarctica. Journal of Quaternary Science 20, 435-446.

März, C., Vogt, C., Schnetger, B., Brumsack, H.-J., 2011. Variable Eocene-Miocene sedimentation processes and bottom water redox conditions in the Central Arctic Ocean (IODP Expedition 302). Earth and Planetary Science Letters 310, 526-537.

McKay, R.M., Browne, G.H., Carter, L., Cowan, E.A., Dunbar, G.B., Krissek, L.A., Naish, T.R., Powell, R.D., Reed, J.A., Wilch, T.I., ANDRILL MIS Science team, 2009. The stratigraphic signature of Late Cenozoic oscillations of the West Antarctic Ice Sheet in Ross Embayment. Geological Society of America Bulletin 121, 1537-1561.

McManus, J., Berelson, W.M., Klinkhammer, G.P., Johnson, K.S., Coale, K.H., Anderson, R.F., Kumar, N., Burdige, D.J., Hammond, D.E., Brumsack, H.J., Mccorkle, D.C. Rushdi, A., 1998a. Geochemistry of barium in marine sediments: implications for its use as a paleoproxy. Geochimica et Cosmochimica Acta 62, 3453-3473.

McManus, J., Berelson, W.M., Klinkhammer, G.P., Kilgore, T.E., Hammond, D.E., 1998b. Remobilization of barium in continental margin sediments. Geochimica et Cosmochimica Acta 58, 4899-4908.

McManus, J., Berelson, W.M., Hammond, D.E., Klinkhammer, G.P., 1999. Barium cycling in the North Pacific: implication for the utility of Ba as a paleoproductivity and paleoalkalinity proxy. Paleoceanography $14,62-73$.

Monien, D., Kuhn, G., von Eynatten, H., Talarico, F.M., 2012. Geochemical provenance analysis of fine-grained sediment revealing Late Miocene to recent PaleoEnvironmental changes in the Western Ross Sea, Antarctica. Global and Planetary Change 96-97, 41-58. http://dx.doi.org/10.1016/j.gloplacha.2010.05.001.

Morford, J.L., Emerson, S., 1999. The geochemistry of redox sensitive trace metals in sediments. Geochimica et Cosmochimica Acta 63, 1735-1750.

Morse, J.W., Luther III, G.W., 1999. Chemical influences on trace metal-sulfide interactions in anoxic sediments. Geochimica et Cosmochimica Acta 63 , 3373-3378.

Müller, P.J., Schneider, R., 1993. An automated leaching method for the determination of opal in sediments and particulate matter. Deep Sea Research Part I: Oceanographic Research Papers 40, 425-444.

Naish, T.R., Levy, R.H., Powell, R.P., the ANDRILL-MIS Science Operations Teams, 2006 ANDRILL McMurdo Ice Shelf Scientific Logistical Implementation Plan. ANDRILL Contribution No. 7.University of Nebraska, Lincoln (117 pp.).
Naish, T., Powell, R., Levy, R., Henrys, S., Krissek, L., Niessen, F., Pompilio, M., Scherer, R., Wilson, G., the ANDRILL-MIS Science Team, 2007. Synthesis of the initial scientific results of the MIS Project (AND-1B Core), Victoria Land Basin, Antarctica. Terra Antarctica 14, 317-327.

Naish, T., Powell, R., Levy, R., Wilson, G., Scherer, R., Talarico, F., Krissek, L., Niessen, F., Pompilio, M., Wilson, T., Carter, L., De Conto, R., Huybers, P., Mckay, R., Pollard, D., Ross, J., Winter, D., Barret, P., Browne, G., Cody, R., Cowan, E., Crampton, J., Dunbar, G., Dunbar, N., Florindo, F., Gebhardt, C., Graham, I., Hannah, M., Hansaraj, D., Harwood, D., Helling, D., Henrys, S., Hinnov, L., Kuhn, G., Kyle, P., Läufer, A., Maffioli, P., Magens, D., Mandernack, K., McIntosh, W., Millan, C., Morin, R., Ohneiser, C., Paulsen, T., Persico, D., Raine, I., Reed, J., Riesselman, C., Sagnotti, L., Schmitt, D., Sjunneskog, C., Strong, P., Taviani, M., Vogel, S., Wilch, T., Williams, T., 2009. Obliquity-paced Pliocene West Antarctic ice sheet oscillations. Nature 458, 322-329. http://dx.doi.org/10.1038/nature07867.

Paytan, A., Kastner, M., Chavez, F.P., 1996. Glacial to interglacial fluctuations in productivity in the Equatorial Pacific as indicated by marine barite. Science 274, 1355-1357.

Paytan, A., Kastner, M., Campbell, D., Thiemens, M.H., 2004. Seawater sulfur isotope fluctuations in the cretaceous. Science 304, 1663-1665.

Piper, D.Z., Perkins, R.B., 2004. A modern vs. Permian black shale - the hydrography, primary productivity, and water-column chemistry of deposition. Chemical Geology 206, 177-197.

Pollard, D., DeConto, R.M., 2009. Modelling West Antarctic ice sheet growth and collapse through the past five million years. Nature 458, 329-333. http://dx.doi.org/10.1038/ nature07809.

Pompilio, M., Dunbar, N., Gebhardt, A.C., Helling, D., Kuhn, G., Kyle, P., Mckay, R., Talarico, F., Tulaczyck, S., Vogel, S., Wilch, T., the ANDRILL-MIS Science Team, 2007. Petrology and geochemistry of the AND-1B Core, Andrill McMurdo Ice Shelf Project. Terra Antarctica 14, 255-288.

Raiswell, R., Fisher, Q.J., 2004. Rates of carbonate cementation associated with sulphate reduction in DSDP/ODP sediments: implications for the formation of concretions. Chemical Geology 211, 71-85

Raiswell, R., Benning, L.G., Tranter, M., Tulaczyk, S., 2008. Bioavailable iron in the Southern Ocean: the significance of the iceberg conveyor belt. Geochemical Transactions 9,7 .

Riedinger, N., Kasten, S., Gröger, J., Franke, C., Pfeifer, K., 2006. Active and buried authigenic barite fronts in sediments from the Eastern Cape Basin. Earth and Planetary Science Letters 241, 876-887.

Rock, N.M.S., 1988. Numerical Geology. Lecture Notes in Earth Sciences 18.SpringerVerlag, New York, Berlin, Heidelberg.

Sanchez-Vidal, A., Collier, R.W., Calafat, A., Fabres, J., Canals, M., 2005. Particulate barium fluxes on the continental margin: a study from the Alboran Sea (western Mediterranean). Marine Chemistry 93, 105-117.

Schimmield, G.B., 1992. Can sediment geochemistry record changes in costal upwelling palaeoproductivity? Evidence from Northwest Africa and the Arabian Sea. In: Summerhayes, C.P., Prell, W.L., Emeis, K.-C. (Eds.), Upwelling systems: evolution since the early Miocene, 28. Geological Society Special Publication, pp. 29-46.

Scopelliti, G., Bellanca, A., Neri, R., Baudin, F., Coccioni, R., 2006. Comparative highresolution chemostratigraphy of the Bonarelli Level from the reference Bottaccione section (Umbria-Marche Apennines) and from an equivalent section in NW Sicily: consistent and contrasting responses to the OAE2. Chemical Geology 228, 266-285.

Scopelliti, G., Neri, R., Bellanca, A., Di Stefano, P., Barbieri, M., 2009. Sedimentology, petrography and geochemistry of a limestone breccia (Pietra di Billiemi) from NW Sicily, Italy: implications for the evolution of the Tethyan basins around the Triassic/Jurassic boundary. Sedimentology 56, 591-607. http://dx.doi.org/10.1111/ j.1365-3091.2008.00987.x.

Scopelliti, G., Bellanca, A., Neri, R., Sabatino, N., 2010. Phosphogenesis in the Bonarelli Level in northwestern Sicily, Italy: petrographic evidence of microbial mediation and related REE behaviour. Cretaceous Research 31, 237-248.

Scopelliti, G., Bellanca, A., Neri, R., 2011. Petrography and carbonate isotope stratigraphy from MIS AND-1B core, Antarctica: evidence of the early Pliocene warming event. Global and Planetary Change 76, 22-32. http://dx.doi.org/10.1016/ j.gloplacha.2010.11.006

Sedwick, P.N., DiTullio, G.R., 1997. Regulation of algal blooms in Antarctic shelf waters by the release of iron from melting sea ice. Geophysical Research Letters 24, 2515-2518.

Sedwick, P.N., DiTullio, G.R., Mackey, D.J., 2000. Iron and manganese in the Ross Sea, Antarctica: seasonal iron limitation in Antarctic shelf waters. Journal of Geophysical Research 105, 11321-11336.

Sternberg, E., Tang, D., Ho, T.-Y., Jeandel, C., Morel, F.M.M., 2005. Barium uptake and adsorption in diatoms. Geochimica et Cosmochimica Acta 69, 2745-2752.

Stickley, C.E., Pike, J., Leventer, A., Dunbar, R., Domack, E.W., Brachfeld, S., Manley, P., McClennan, C., 2005. Deglacial ocean and climate seasonality in laminated diatom sediments, MacRobertson Shelf, Antarctica. Palaeogeography, Palaeoclimatology, Palaeoecology 227, 290-310.

Torres, M.E., Brumsack, H.J., Bohrmann, G., Emeis, K.C., 1996. Barite fronts in continental margin sediments: a new look at barium remobilization in the zone of sulfate reduction and formation of heavy barites in diagenetic fronts. Chemical Geology 127, 125-139.

Tribovillard, N., Algeo, T.J., Lyons, T., Riboulleau, A., 2006. Trace metals as paleoredox and paleoproductivity proxies: an update. Chemical Geology 232, 12-32.

van Lith, Y., Warthmann, R., Vasconcelos, C., McKenzie, J.A., 2003. Microbial fossilization in carbonate sediments: a result of the bacterial surface involvement in dolomite precipitation. Sedimentology 50, 237-245. 
Vasconcelos, C., McKenzie, J.A., 1997. Microbial mediation of modern dolomite precipitation and diagenesis under anoxic conditions (Lagoa Vermelha, Rio de Janeiro, Brazil). Journal of Sedimentary Research 67, 378-390.

Vinogradova, Z.A., Koval'skiy, V.V., 1962. Elemental composition of the Black Sea plankton. Doklady Akademii Nauk SSSR 147, 217-219.

von Breymann, M.T., Brumsack, H., Emeis, K.-C., 1992. Depositional and diagenetic behavior of barium in the Japan Sea. In: Pisciotto, K.A., Ingle, J.C., von Breymann, M.T., Barron, J., et al. (Eds.), Proceedings of ODP, Scientific Results, 127/128. College Station, Texas, USA, pp. 651-663.

Wanty, R.B., Goldhaber, R., 1992. Thermodynamics and kinetics of reactions involving vanadium in natural systems: accumulation of vanadium in sedimentary rock. Geochimica et Cosmochimica Acta 56, 171-183.
Warthmann, R., van Lith, Y., Vasconcelos, C., McKenzie, J.A., Karpoff, A.M., 2000 Bacterially induced dolomite precipitation in anoxic culture experiments. Geology 28, 1091-1094.

Wehrly, B., Stumm, W., 1989. Vanadyl in natural waters: adsorption, and hydrolysis promote oxygenation. Geochimica et Cosmochimica Acta 53, 69-77.

Winter, D., Siunneskog, C., Harwood, D., 2010. Early to mid-Pliocene environmentally constrained diatom assemblages from the AND-1B drill core, McMurdo Sound Antarctica. Stratigraphy 2010, 207-227.

Winter, D., Siunneskog, C., Scherer, R., Maffioli, P., Harwood, D., 2012. Diatom-based correlation of early to mid-Pliocene drill cores from the southwestern Ross Sea Antarctica. Global and Planetary Change 96-97, 131-142. http://dx.doi.org/ 10.1016/j.gloplache.2009.12.004 